\title{
Head and neck squamous cell carcinoma
}

Daniel E. Johnson', Barbara Burtness' ${ }^{2}$, C. René Leemans ${ }^{3}$, Vivian Wai Yan Lui', Julie E. Bauman ${ }^{5}$ and Jennifer R. Grandis ${ }^{1 凶}$

Abstract | Most head and neck cancers are derived from the mucosal epithelium in the oral cavity, pharynx and larynx and are known collectively as head and neck squamous cell carcinoma (HNSCC). Oral cavity and larynx cancers are generally associated with tobacco consumption, alcohol abuse or both, whereas pharynx cancers are increasingly attributed to infection with human papillomavirus (HPV), primarily HPV-16. Thus, HNSCC can be separated into HPV-negative and HPV-positive HNSCC. Despite evidence of histological progression from cellular atypia through various degrees of dysplasia, ultimately leading to invasive HNSCC, most patients are diagnosed with late-stage HNSCC without a clinically evident antecedent pre-malignant lesion. Traditional staging of HNSCC using the tumour-node-metastasis system has been supplemented by the 2017 AJCC/UICC staging system, which incorporates additional information relevant to HPV-positive disease. Treatment is generally multimodal, consisting of surgery followed by chemoradiotherapy (CRT) for oral cavity cancers and primary CRT for pharynx and larynx cancers. The EGFR monoclonal antibody cetuximab is generally used in combination with radiation in HPV-negative HNSCC where comorbidities prevent the use of cytotoxic chemotherapy. The FDA approved the immune checkpoint inhibitors pembrolizumab and nivolumab for treatment of recurrent or metastatic HNSCC and pembrolizumab as primary treatment for unresectable disease. Elucidation of the molecular genetic landscape of HNSCC over the past decade has revealed new opportunities for therapeutic intervention. Ongoing efforts aim to integrate our understanding of HNSCC biology and immunobiology to identify predictive biomarkers that will enable delivery of the most effective, least-toxic therapies.

Head and neck squamous cell carcinomas (HNSCCs) develop from the mucosal epithelium in the oral cavity, pharynx and larynx and are the most common malignancies that arise in the head and neck (FIG. 1). The burden of HNSCC varies across countries/regions and has generally been correlated with exposure to tobacco-derived carcinogens, excessive alcohol consumption, or both. Increasingly, tumours that arise in the oropharynx are linked to prior infection with oncogenic strains of human papillomavirus (HPV), primarily HPV-16, and, to a lesser extent, HPV-18 and other strains ${ }^{1-3}$. As the most common oncogenic HPVs, HPV-16 and HPV-18, are covered by FDA-approved HPV vaccines, it is feasible that HPV-positive HNSCC could be prevented by successful vaccination campaigns worldwide. HNSCCs of the oral cavity and larynx are still primarily associated with smoking and are now collectively referred to as HPV-negative HNSCC. No screening strategy has proved to be effective, and careful physical examination remains the primary approach for early detection.
Although a proportion of oral pre-malignant lesions (OPLs), which present as leukoplakia (white patches) or erythroplakia (red patches), progress to invasive cancer, the majority of patients present with advancedstage HNSCC without a clinical history of a pre-malignancy. HNSCC of the oral cavity is generally treated with surgical resection, followed by adjuvant radiation or chemotherapy plus radiation (known as chemoradiation or chemoradiotherapy (CRT)) depending on the disease stage. CRT has been the primary approach to treat cancers that arise in the pharynx or larynx. HPV-positive HNSCC generally has a more favourable prognosis than HPV-negative HNSCC, and ongoing studies are testing the efficacy of therapeutic dose reduction (of both radiation and chemotherapy) in HPV-positive disease treatment. With the exception of early-stage oral cavity cancers (which are treated with surgery alone) or larynx cancers (which are amenable to surgery or radiation alone), treatment of the majority of patients with HNSCC requires multimodality approaches and 


\author{
Author addresses \\ ${ }^{1}$ Department of Otolaryngology-Head and Neck Surgery, University of California at \\ San Francisco, San Francisco, CA, USA. \\ 2Department of Medicine, Yale University School of Medicine and Yale Cancer Center, \\ New Haven, CT, USA. \\ ${ }^{3}$ Department of Otolaryngology-Head and Neck Surgery, Amsterdam University Medical \\ Center, Vrije Universiteit Amsterdam, Cancer Center Amsterdam, Amsterdam, Netherlands. \\ ${ }^{4}$ School of Biomedical Sciences, Faculty of Medicine, The Chinese University of \\ Hong Kong, Hong Kong, Hong Kong SAR. \\ ${ }^{5}$ Department of Medicine-Hematology/Oncology, University of Arizona, Tucson, AZ, USA.
}

thus multidisciplinary care. The epidermal growth factor receptor (EGFR; also known as HER1) monoclonal antibody cetuximab is approved by the FDA as a radiation sensitizer, alone or in combination with chemotherapy, for the treatment of patients with recurrent or metastatic disease ${ }^{4}$. Although inferior to cisplatin as a radiosensitizer in $\mathrm{HPV}$-associated disease ${ }^{5,6}$, cetuximab is often used in cisplatin-ineligible patients. The immune checkpoint inhibitors pembrolizumab and nivolumab are approved by the FDA for treatment of cisplatin-refractory recurrent or metastatic HNSCC and pembrolizumab is approved as first-line therapy in patients who present with unresectable or metastatic disease $^{7-9}$. Detailed molecular characterization as well as immune profiling of HNSCC suggests that incorporation of prognostic and predictive biomarkers into clinical management may overcome obstacles to targeted therapies and enable prolonged survival. In this Primer, we provide an overview of the types of HNSCC and their epidemiology, as well as the pathogenesis of each type and how this influences the management approach.

\section{Epidemiology Incidence, prevalence and mortality}

HNSCC is the sixth most common cancer worldwide, with 890,000 new cases and 450,000 deaths in 2018 (REFS $^{10-12}$ ) (FIG. 2). The incidence of HNSCC continues to rise and is anticipated to increase by $30 \%$ (that is, 1.08 million new cases annually) by 2030 (Global Cancer Observatory (GLOBOCAN) $)^{10-12}$. The high prevalence of HNSCC in regions such as Southeast Asia and Australia is associated with consumption of specific carcinogen-containing products (described below), whereas increasing rates of oropharyngeal infection with HPV have contributed to the high prevalence of HNSCC in the USA and Western Europe ${ }^{13-15}$. In general, men are at twofold to fourfold higher risk than women for developing HNSCC. The median age of diagnosis for non-virally associated HNSCC is 66 years, whereas the median age of diagnosis for HPV-associated oropharyngeal cancer and Epstein-Barr virus (EBV)-associated nasopharyngeal cancer is $\sim 53$ years and $\sim 50$ years, respectively ${ }^{16,17}$. The survival for HNSCC has improved modestly over the past three decades; for example, the 5 -year survival increased from $55 \%$ during the period $1992-1996$ to $66 \%$ during the period 2002-2006 when analysed across all age groups and anatomical sites within the Surveillance, Epidemiology, and End Results (SEER) registry ${ }^{18}$. A subgroup analysis showed improved survival in all age groups except in older patients ( $>75$ years of age) and for all anatomical sites except the larynx, where survival was stagnant. Improvement in survival is partially attributable to the emergence of HPV-associated HNSCC, a population with improved prognosis, rather than improvements in multimodality treatment per se; a subsequent SEER analysis incorporating tissue assessment for HPV noted improved survival in patients with HPV-positive HNSCC but not in those with HPV-negative HNSCC ${ }^{19}$. In addition to deaths directly caused by HNSCC, survivors of this cancer have the second highest rate of suicide (63.4 cases per 100,000 individuals) after those with pancreatic cancer (86.4 cases per 100,000 individuals), compared with survivors of other cancers (23.6 cases per 100,000 individuals). Psychological distress and compromised quality of life (QOL) are likely key underlying factors for suicide ${ }^{20}$.

\section{Risk factors}

Epidemiological studies have revealed a diverse range of risk factors for HNSCC, as classified by the International Agency for Research on Cancer (IARC) of the World Health organization $(\mathrm{WHO})^{21}$. These risk factors include tobacco consumption, alcohol consumption, exposure to environmental pollutants and infection with viral agents, namely, HPV and EBV. Interestingly, several risk factors display geographical or cultural and/or habitual prevalence. Tobacco and alcohol consumption are the high-risk factors that occur most widely geographically. Of note, heavy users of both substances have a $>35$-fold higher risk of developing $\mathrm{HNSCC}^{22}$. Among some AsiaPacific populations, oral cavity cancer is associated with chewing of areca nut products including 'betel quid', a term that applies to a variety of customized mixtures comprising areca nut (Areca catechu; the carcinogen source), betel leaf (the leaf of Piper betle), slaked lime and/or tobacco, as well as spices according to local custom $^{21}$. The use of areca nut or betel quid products is linked to particularly high rates of oral cavity cancer in India (first and fourth most common cancer in Indian men and women, respectively), Taiwan and some provinces in mainland $\mathrm{China}^{23}$. In general, the high male to female ratios for HPV-negative HNSCC incidence reflect the sex-specific patterns of modifiable risk behaviours, including the use of the aforementioned tobacco, smokeless tobacco, areca nut, betel quid and alcohol ${ }^{21,24}$.

The effect of electronic cigarettes on HNSCC risk remains unknown and will only be evident in the coming decades. Exposure to carcinogenic air pollutants, including organic and inorganic chemicals, as well as particulate matter, is a risk factor for HNSCC, especially in developing countries/regions with worsening air pollution, such as India and China ${ }^{25,26}$. Other risk factors include ageing, poor oral hygiene and diets lacking in vegetables $^{27,28}$. In terms of infectious agents, persistent infection with HPV and EBV are known aetiological risk factors for HNSCC arising from the oropharynx and nasopharynx, respectively ${ }^{29,30}$. The male to female ratio for HPV-positive HNSCC incidence ranges from three to $\operatorname{six}^{31}$, which is explained by higher rates of persistent oropharyngeal HPV infection among men despite similar prevalences of anogenital HPV infection ${ }^{32-34}$. HPV infection that leads to HNSCC is mainly transmitted by oral sex, and the incidence of HPV-positive HNSCC 

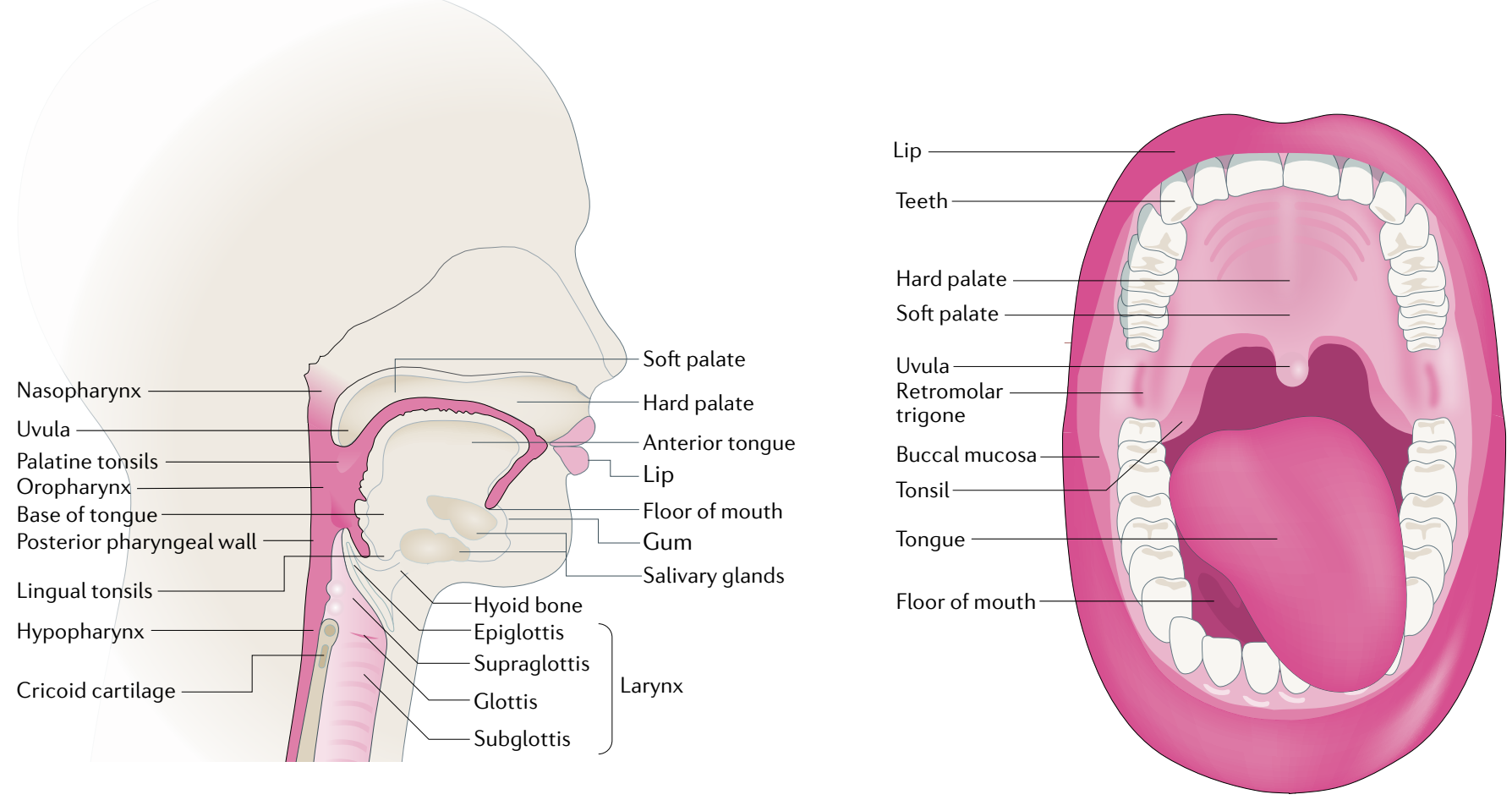

Fig. 1 | Anatomical sites of HNSCC development. Head and neck squamous cell carcinoma (HNSCC) arises from the mucosal epithelium of the oral cavity (lips, buccal mucosa, hard palate, anterior tongue, floor of mouth and retromolar trigone), nasopharynx, oropharynx (palatine tonsils, lingual tonsils, base of tongue, soft palate, uvula and posterior pharyngeal wall), hypopharynx (the bottom part of the throat, extending from the hyoid bone to the cricoid cartilage) and larynx. Human papillomavirus-associated HNSCCs arise primarily from the palatine and lingual tonsils of the oropharynx, whereas tobacco-associated HNSCCs arise primarily in the oral cavity, hypopharynx and larynx.

continues to rise, especially in populations that are not vaccinated against HPV prior to HPV exposure ${ }^{35,36}$.

In addition, genetic factors also contribute to HNSCC risk. Individuals with Fanconi anaemia, a rare, inherited genetic disease characterized by impaired DNA repair (owing to mutations in any of the 22 FANC genes), have a 500-700-fold increased risk of developing HNSCC, primarily cancers of the oral cavity ${ }^{37}$. Although the mechanisms responsible for the unique proclivity of patients with Fanconi anaemia to develop HNSCC remain unknown, alterations in Fanconi anaemia pathway genes are likely to have a role. Meta-analyses have shown that polymorphisms in genes involved in carcinogen metabolism and in immunity are associated with increased risk, including polymorphisms in CTLA4 (rs231775 and rs4553808), Il10 (1082A $>$ G), cytochrome P450 1A1 (CYP1A1; Ile462Val) and glutathione $S$-transferase $\mu 1$ (GSTM1; null polymorphism) $)^{38-41}$. Thus, a reduced ability to metabolize carcinogens and weakened immunity may contribute to HNSCC. Decreased use of tobacco products, improved oral health and widespread HPV vaccination should help reduce the global incidence of $\mathrm{HNSCC}^{42}$.

\section{Mechanisms/pathophysiology}

Formation, progression and cell of origin

HNSCC originates from mucosal epithelial cells that line the oral cavity, pharynx, larynx and sinonasal tract. Histologically, progression to invasive HNSCC follows an ordered series of steps beginning with epithelial cell hyperplasia, followed by dysplasia (mild, moderate and severe), carcinoma in situ and, ultimately, invasive carcinoma (FIG. 3). However, of note, most patients diagnosed with HNSCC do not have a history of an antecedent pre-malignant lesion. Given the heterogeneous nature of HNSCC, the cell of origin depends on anatomical location and aetiological agent (carcinogen versus virus). However, in each case, normal adult stem or progenitor cells are likely candidates for the cell of origin, giving rise, following oncogenic transformation, to cancer stem cells (CSCs) with properties of self-renewal and pluripotency. HNSCC CSCs with the capacity to generate tumours in transplantation assays constitute only a minor fraction (1-3\%) of the cells in primary tumours ${ }^{43}$ but, despite their inherent resistance to conventional drugs, represent attractive targets for novel targeting agents.

A number of molecular biomarkers of HNSCC CSCs have been proposed, with CD44, CD133 and ALDH1 being the most extensively validated and associated with prognostic significance. CD44 is a cell surface receptor for hyaluronic acid and matrix metalloproteinases (MMPs) and is involved in intercellular interactions and cell migration. HNSCC cells with high levels of CD44 are capable of self-renewal, and CD44 levels in HNSCC tumours are associated with metastasis and a poor prognosis ${ }^{44,45}$. Similarly, increased levels of the membrane-spanning protein CD133 are associated with HNSCC invasiveness and metastasis ${ }^{46}$. ALDH1 is 


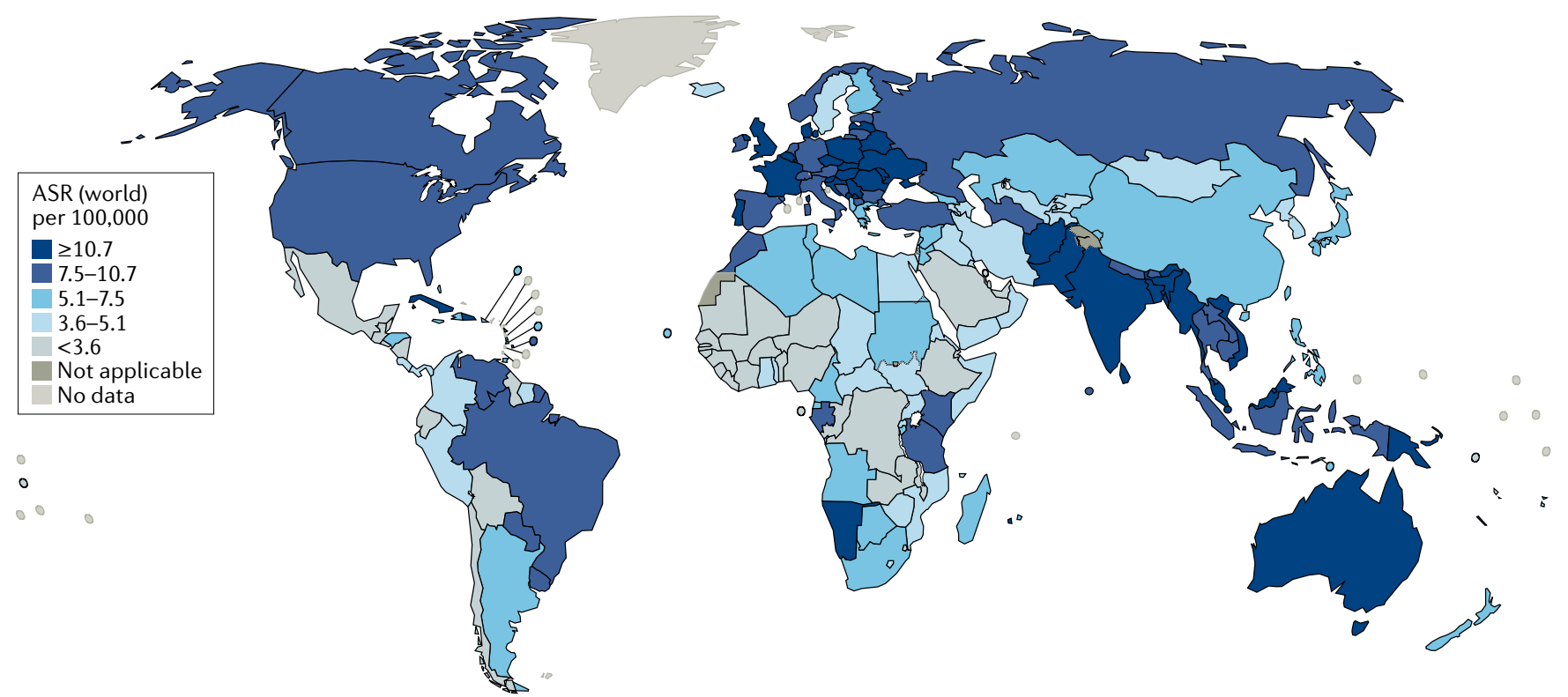

Fig. 2 | Global incidence of HNSCC. The estimated age-standardized rates (ASRs) of head and neck squamous cell carcinoma (HNSCC) incidence worldwide are shown for men and women combined ${ }^{10-12}$. The data are from GLOBOCAN for $2018\left(\right.$ REF. $^{10}$ ). The map was generated using the GLOBOCAN website mapping tool by selecting the 'hypopharynx', 'larynx', 'lip, oral cavity', 'nasopharynx' and 'oropharynx' cancer sites.

an intracellular enzyme that converts retinol into retinoic acid, plays a part in cellular detoxification and is a marker for both normal stem cells and CSCs. High levels of ALDH1 expression or activity are associated with self-renewal, invasion and metastasis and may have prognostic significance in $\mathrm{HNSCC}^{45}$. In addition, HNSCC cells with CSC properties express elevated levels of the stem cell markers OCT3, OCT4, SOX2 and NANOG, with the levels of these proteins correlating with tumour grade in oral cancers ${ }^{47}$. Immunohistochemical analyses of HNSCC tumours indicate that $\sim 80 \%$ of $\mathrm{ALDH}^{+}$cells are in close proximity $(\leq 100 \mu \mathrm{m})$ to a blood vessel, suggesting that the CSCs reside primarily in perivascular niches ${ }^{43}$.

An important clinical phenomenon to consider when defining the cell of origin in HNSCC is the development of second primary tumours (SPTs). Synchronous and/or metachronous SPTs arise at an extraordinarily high rate after the diagnosis of an initial primary tumour and can be localized at distinct anatomical sites in the head and neck region, oesophagus or lungs ${ }^{48,49}$. Frequently lethal, SPTs may share some molecular abnormalities with the initial primary tumour or may exhibit marked differences. The concept of 'field cancerization' suggests that carcinogens damage or condemn large anatomical fields ${ }^{50}$. In tobacco-associated HNSCC, the size of the damaged anatomical field may increase with patient age $^{51,52}$. Considering the concept of field cancerization, the development of SPTs may reflect distinct CSCs arising from independent oncogenic transformations.

\section{Initiating and early events}

HPV-negative HNSCC. Tobacco consumption is the primary risk factor for development of HPV-negative HNSCC. Tobacco consists of more than 5,000 different chemicals, of which dozens have been shown to have carcinogenic activity. The chemicals thought to be most responsible for the cancer-causing effects of tobacco are polycyclic aromatic hydrocarbons (PAHs), including benzo(a)pyrene, and nitrosamines, including 4-(methylnitrosamine)-1-(3-pyridyl)-1-butanone and $N$-nitrosonornicotine ${ }^{53,54}$. In smokeless tobacco, nitrosamines are the dominant carcinogen, whereas the carcinogens in areca nut and betel quid are poorly defined ${ }^{55}$. Tobacco-derived carcinogens, including PAHs and nitrosamines, undergo metabolic activation, with detoxification enzymes and pathways promoting excretion (FIG. 4). However, many of the reactive metabolites of these carcinogens can also form covalent DNA adducts, which, if not properly repaired, lead to mutations and other genetic abnormalities. The propensity for tobacco carcinogens to promote genetic changes and neoplastic transformation probably depends on the balance between metabolic activation versus detoxification and DNA repair (FIG. 4). The use of tobacco products is also associated with inflammation in the exposed tissues. Coincident with inflammation is the local production of cytokines, chemokines and growth factors that can have an important role in promoting proliferation, angiogenesis and, ultimately, carcinogenesis.

Excessive alcohol consumption is another key risk factor for HPV-negative HNSCC and is known to synergize with tobacco use to promote carcinogenesis ${ }^{56}$. Alcohol might serve as a solvent for carcinogens, enhancing the exposure of epithelial cells to these substances ${ }^{57}$. In addition, alcohol is metabolized to acetaldehyde, which is known to form DNA adducts ${ }^{58}$.

HPV-positive HNSCC. Infection with HPV is an increasingly common risk factor for HNSCC. HPV infection is associated with most oropharyngeal cancers $(>70 \%)$ and a small minority of cancers at other anatomical sites in the head and neck ${ }^{1,2}$. As described 
below, HPV-positive HNSCC exhibits distinct differences from HPV-negative HNSCC in gene expression, and mutational and immune profiles, underscoring the unique biology of this disease. Infection with HPV is an early event in HPV-positive HNSCC, and most of these cancers arise from deep crypts in the palatine and lingual tonsils (FIG. 5). HPV-16 is the primary causative type, although other high-risk HPVs, including HPV-18, HPV-31, HPV-33 and HPV-52, are detected in a small percentage of patients ${ }^{3}$. The high-risk HPVs, including HPV-16, are small, double-stranded, circular DNA viruses with a genome of $\sim 8 \mathrm{~kb}$. In HNSCC tumour specimens, the viral genome is typically found integrated at a single, albeit variant, genomic site ${ }^{59}$. The genome consists of seven early genes $(E 1-E 7)$ and two late genes ( $L 1$ and $L 2$ ). The $L 1$ and $L 2$ genes encode viral capsid proteins, whereas the E1-E5 genes encode proteins that are primarily involved in replication and transcription of the viral genome. The bicistronic E6 and E7 genes are essential for oncogenic transformation of the host cell. E6 protein forms a complex with the cellular ubiquitylation protein E6-AP and the tumour suppressor $\mathrm{p} 53$ to promote ubiquitylation and proteasomal degradation of p53 (REF. $\left.{ }^{60}\right)$. In contrast to HPV-negative HNSCC, in which TP53 (encoding p53) is frequently deleted or mutated, this gene is rarely altered in HPV-positive HNSCC, as p53 is eliminated by the action of E6 (REF. ${ }^{59}$ ). E6 may possess other transforming activities beyond $\mathrm{p} 53$ degradation but these functions are less well characterized ${ }^{61-63}$. E7 protein binds strongly to the cell cycle regulator retinoblastoma-associated protein (RB1), promoting proteasomal destruction of $\mathrm{RB} 1$ and the release of E2F family transcription factors ${ }^{64}$. The liberated E2F proteins drive the cell cycle beyond the restriction point (also known as the G1-S checkpoint) and into $S$ phase. E7 also interacts with and affects the levels and/or cellular activity of a number of other cell cycle regulatory proteins $\mathrm{s}^{62,65}$. The disruption of RB1 function by E7 leads to a feedback upregulation of $\mathrm{p} 16^{\mathrm{INK} 4 \mathrm{~A}}$, and detection of $\mathrm{p} 16^{\mathrm{INK} 4 \mathrm{~A}}$ expression is commonly used to classify oropharyngeal tumours as
HPV-positive. In addition to E6 and E7, E5 also has a role in oncogenic transformation by helping to drive cell cycle progression ${ }^{66,67}$.

\section{Genomic alterations and key pathways}

There is a tremendous need to identify molecular biomarkers that can be used to predict progression of pre-malignant HNSCC lesions, prognosticate survival, reveal new targets for intervention and predict response to therapeutic agents. The search for biomarkers has focused on defining the molecular abnormalities that characterize HNSCC. In this section, we highlight findings regarding genetic and epigenetic alterations, as well as dysregulation of cellular signalling pathways, which occur during HNSCC development.

HNSCC is characterized by genetic instability, with frequent loss or gain of chromosomal regions ${ }^{59}$. The availability of a model of ordered histological progression of HNSCC has enabled assignment of some chromosomal abnormalities to specific stages of progression ${ }^{57,68}$ (FIG. 3). Loss of 9p21 occurs during progression of normal head and neck epithelial mucosa to hyperplasia. The $9 \mathrm{p} 21$ region includes the tumour suppressor genes (TSGs) CDKN2A (encoding the CDK4 and CDK6 inhibitor $\mathrm{p} 16^{\mathrm{INK} 4 \mathrm{~A}}$ ) and $A R F$ (encoding p14, a stabilizer of p53). Progression from hyperplasia to dysplasia is marked by loss of $3 \mathrm{p} 21$ and $17 \mathrm{p} 13$, the site of TP53. The transition from dysplasia to carcinoma in situ involves loss of 11q13,13q21 and 14q32, whereas loss of $6 \mathrm{p}, 8,4 \mathrm{q} 27$ and $10 \mathrm{q} 23$ is observed in the progression to invasive carcinoma. Collectively, these studies of chromosomal abnormalities reveal that multiple genetic alterations may be required for full transformation to invasive $\mathrm{HNSCC}^{68}$. Furthermore, whether progression of HNSCC is strictly dependent on the temporal sequence of these alterations or, instead, on their collective accumulation, remains unresolved.

The Cancer Genome Atlas (TCGA) contains a wealth of data on copy number alterations (CNAs), mutational profiles, mRNA expression and microRNA (miRNA) expression from over 520 human HNSCC tumours ${ }^{59}$.

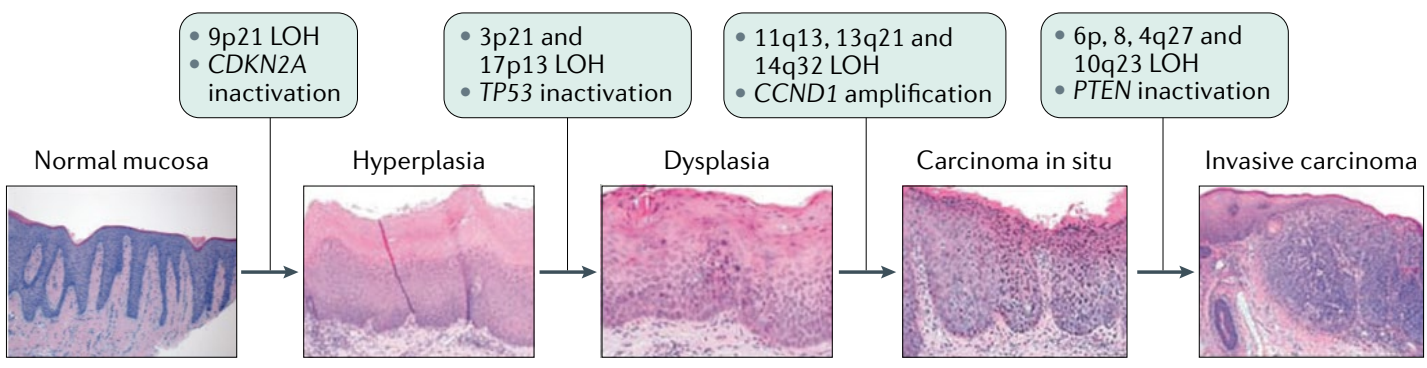

Fig. 3 | Progression of HNSCC and key genetic events. The mucosal epithelium lining the oral cavity, pharynx, larynx and sinonasal tract is the site of origin for head and neck squamous cell carcinoma (HNSCC). In a model of ordered histological progression of $\mathrm{HNSCC}^{68}$, mucosal epithelial cell hyperplasia is followed by dysplasia, and carcinoma in situ precedes the development of invasive carcinoma. Specific genetic events have been found to be enriched at each stage of progression and are indicated. Of note, unlike in most cancers in which oncogenic mutations typically drive tumorigenesis, HNSCC formation usually involves the inactivation of tumour suppressor genes, such as CDKN2A and TP53 (encoding p16 $6^{\text {INK4A }}$ and p53, respectively) in early stages and PTEN (encoding phosphatase and tensin homologue (PTEN)) at later stages. LOH, loss of heterozygosity. Histopathology images of hyperplasia, dysplasia, carcinoma in situ and invasive carcinoma are reprinted from REF. ${ }^{250}$, Springer Nature Limited. Histopathology image of normal mucosa courtesy of R. Jordan, University of California San Francisco. 


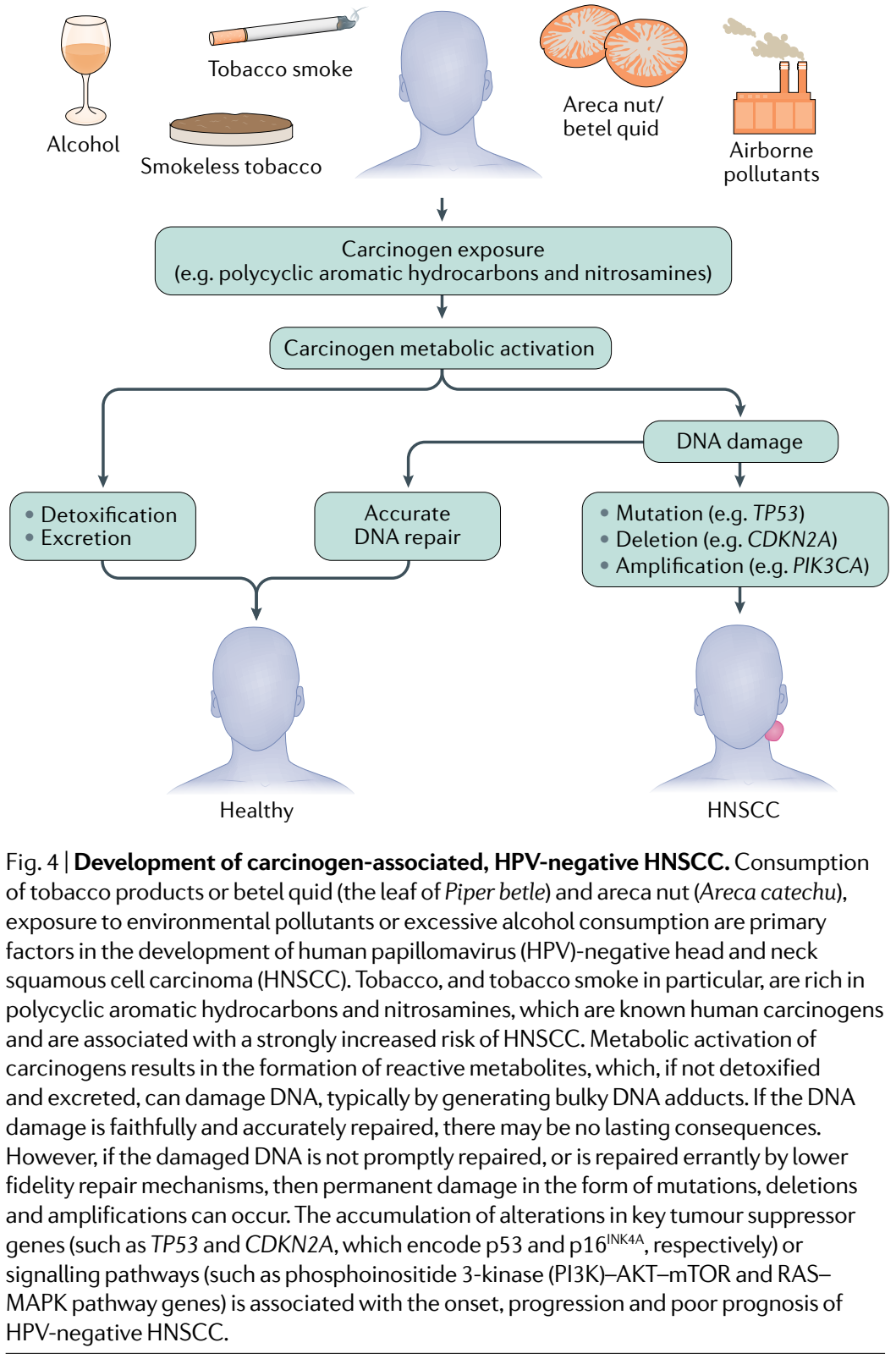

A detailed analysis of 279 of these tumours, consisting of $243 \mathrm{HPV}$-negative and $36 \mathrm{HPV}$-positive tumours, revealed a high degree of genomic instability; tumours had an average of 141 CNAs and 62 chromosomal structural abnormalities (for example, fusions) ${ }^{59}$. HNSCC tumours exhibited frequent mutation of $C D K N 2 A$ ( $22 \%$ of tumours) and TP53 (72\% of tumours). Coupled with the aforementioned highly frequent chromosomal loss of CDKN2A and TP53 in HNSCC tumours, these TSGs are regarded as the most frequently altered genes in HNSCC. However, these alterations are largely restricted to HPV-negative tumours, owing to the action of HPV E6 and E7 proteins in eliminating p53 and RB1 (p16 ${ }^{\mathrm{INK} 4 \mathrm{~A}}$ inhibits phosphorylation of RB1). Mutational profiling revealed that HNSCC-associated mutations are significantly enriched in 11 genes $^{59}$. Interestingly, this list of frequently mutated genes is dominated by known and potential tumour suppressors, including TP53, CDKN2A, FAT1, NOTCH1, KMT2D, NSD1 and
TGFBR2. PIK3CA, encoding the catalytic subunit of phosphoinositide 3-kinase (PI3K), is the only oncogene that is found to be frequently mutated $(\sim 14 \%)$ in HNSCC $^{59,69-72}$. Mutations in RAS genes are infrequent, with HRAS mutations being the most common ( $\sim 4 \%$ of tumours). Thus, in contrast to many other solid tumour malignancies that are frequently driven by mutations in RAS or other oncogenes, HNSCC might be more frequently driven by loss of tumour suppressors. Comparison of HPV-positive and HPV-negative tumours failed to detect previously reported differences in overall mutation rates ${ }^{71}$. However, HPV-positive tumours uniquely show frequent loss of TRAF3 and amplification of E2F1 (whereas HPV-negative tumours show frequent alterations in CDKN2A and TP53), frequent focal deletions in other TSGs (such as NSD1, FAT1, NOTCH1 and SMAD4) and frequent focal amplification of the genes encoding the receptor tyrosine kinases EGFR, HER2 (also known as ERBB2) and FGFR1 (REF. ${ }^{59}$ ). Mutations in the genes encoding NRF2 (NFE2L2) and KEAP1 (KEAP1), key regulators of oxidative stress, are also common and occur exclusively in HPV-negative HNSCC ${ }^{59}$.

Two additional members of the TP53 gene family, TP63 and TP73, are frequently altered in HNSCC. TP63 encodes two major isoforms, $\Delta \mathrm{Np} 63$ and TAp63 (containing a truncated or complete transactivation (TA) domain, respectively), and is overexpressed in a majority of HNSCC tumours ${ }^{73} . \Delta$ Np63 promotes HNSCC tumour growth by multiple mechanisms, including suppression of apoptosis and $\mathrm{p} 16^{\mathrm{INK} 4 \mathrm{~A}}$ expression and induction of mitogenic signalling ${ }^{73-76}$. By contrast, TAp73, a major isoform encoded by TP73, exhibits tumour suppressor activity, and the function of TAp73 is commonly abolished in HNSCC. For example, stimulation of HNSCC cells with TNF results in the induction of REL oncoprotein that binds to $\triangle \mathrm{Np} 63$, displacing TAp73 from $\triangle \mathrm{Np} 63-\mathrm{TAp} 73$ complexes and inactivating TAp73 $\left(\right.$ REFS $\left.^{77,78}\right)$. Phosphorylation of TAp73 by casein kinase 2 or Polo-like kinase 2 also leads to TAp73 inactivation and results in induction of NANOG, SOX2 and OCT4, promoting the stem cell-like properties of HNSCC tumour cells ${ }^{79,80}$.

In addition to genetic alterations, epigenetic changes also have a role in driving HNSCC oncogenesis. Although HNSCC tumours are characterized by global hypomethylation of DNA, hypermethylation and resultant downregulated expression of key TSGs, including $C D K N 2 A, R A R B$ (encoding RAR $\beta$ ), DCC and MGMT, occurs frequently ${ }^{81-83}$. MGMT is notable, as this protein is involved in the repair of DNA damage from tobacco carcinogens, whereas RAR $\beta$ mediates epithelial cell differentiation.

Numerous studies have demonstrated aberrant expression of signalling proteins and/or activation of signalling pathways in HNSCC tumours. EGFR is overexpressed in $80-90 \%$ of HNSCC tumours and is associated with poor overall survival (OS) and progression-free survival ${ }^{84,85}$. Molecular targeting of EGFR with monoclonal antibodies (such as cetuximab) is an FDA-approved strategy for inhibiting EGFR signalling in HNSCC. Overexpression of other receptor tyrosine kinases, 

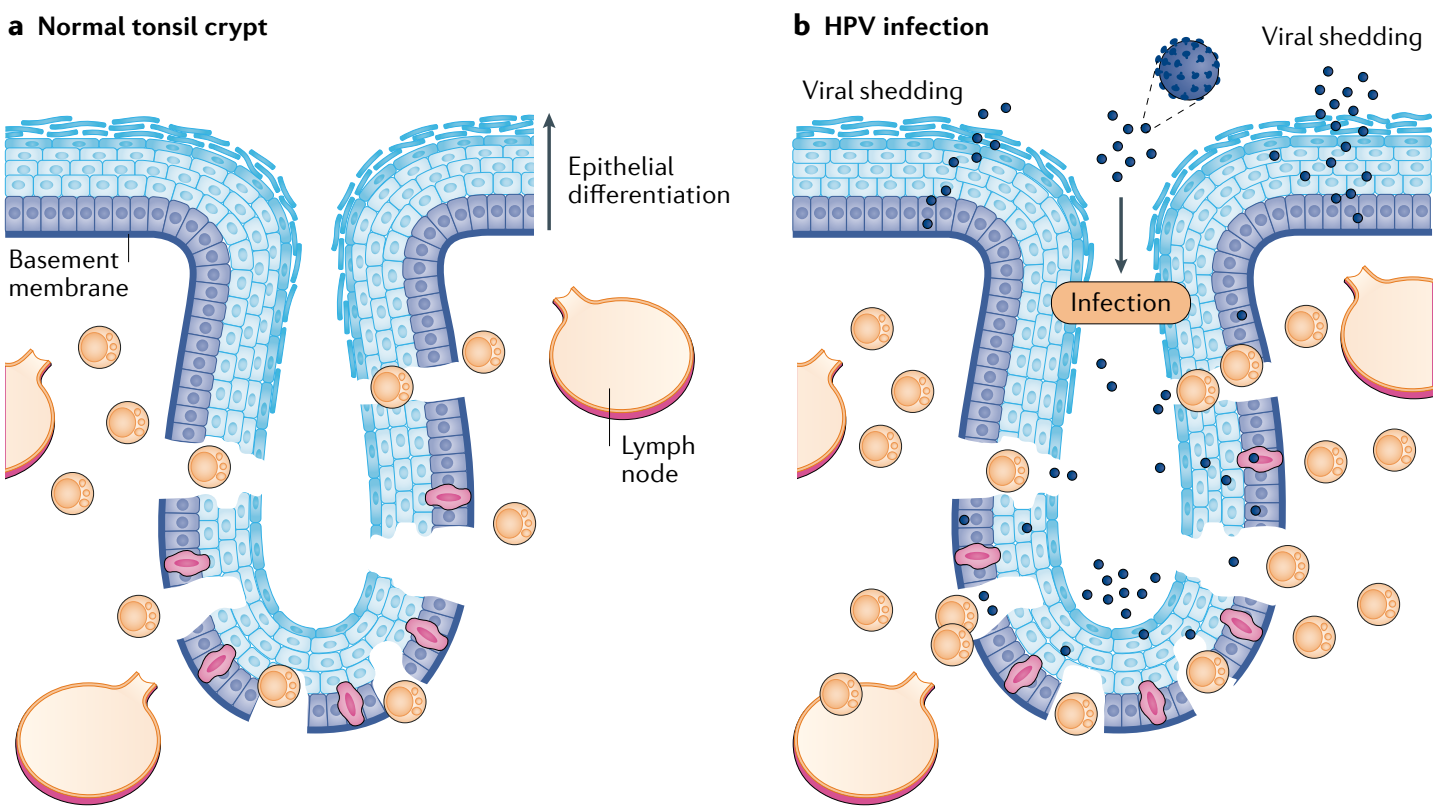

c HPV-mediated malignant transformation
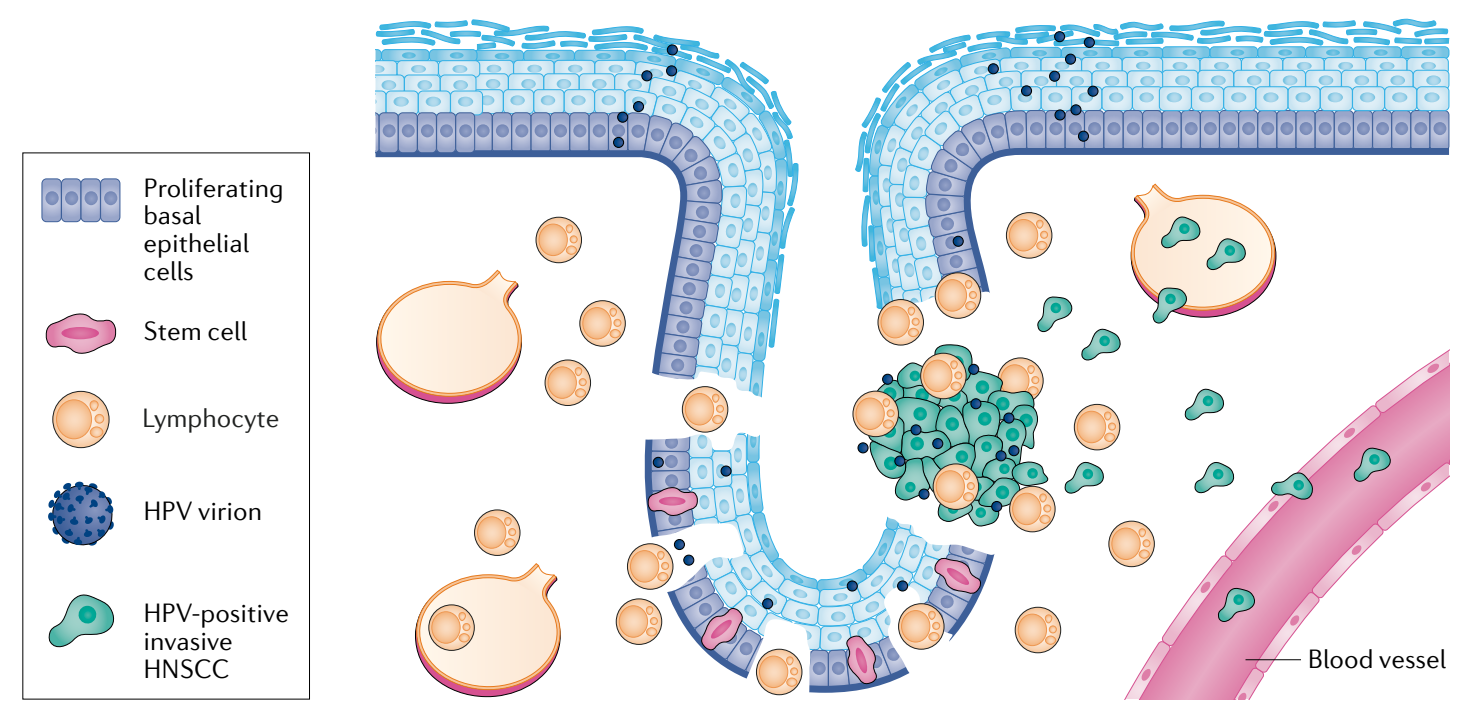

Fig. 5 | HPV infection of the tonsil crypt and development of HPV-positive HNSCC. a | Throughout the tonsil epithelium, proliferating basal epithelial cells constitute the cell layer adjacent to the basement membrane. Differentiation of basal epithelial cells leads to their detachment from the basement membrane and upward migration, with progressively increasing differentiation leading to the sloughing off of terminally differentiated, non-proliferating cells. The palatine and lingual tonsils are also characterized by numerous tissue invaginations, commonly termed crypts, which are particularly enriched in stem cells at their base. A unique, reticulated squamous epithelium lines the crypt structure and gaps or fissures in the basement membrane and basal layer also occur. The presence of these fissures allows lymphocytes to enter the crypts and directly interact with foreign, external antigens. $\mathbf{b}$ | During infection with human papillomavirus (HPV), the reticulated and disrupted nature of the crypt squamous epithelium allows viral access to stem cells, proliferating basal cells and the basement membrane. Infiltrating immune cells also make contact with the viral particles. In the case of a productive infection, distinct viral genes and proteins are induced and/or activated during the different stages of epithelial cell differentiation, culminating in the production and shedding of new viral particles. $\mathrm{c} \mid$ Stem cells or proliferating basal cells represent probable cells of origin for HPV-positive head and neck squamous cell carcinoma (HNSCC). Stable integration of the viral genome into the host genome, and the concerted action of HPV E6 and E7 proteins on cellular p53 and RB levels, respectively, acts to promote cellular transformation. The accumulation of additional genetic alterations is needed to induce full transformation, including the acquisition of invasive and metastatic phenotypes. 
including HER2 and MET, also occurs and may contribute to HNSCC resistance to EGFR-targeting agents ${ }^{73,86,87}$. Overexpression of the cytokine IL- 6 and its receptor is also implicated in the poor prognosis of HNSCC ${ }^{88,89}$. Amplification of CCND1, resulting in overexpression of cyclin D1, is associated with the progression of dysplastic lesions to carcinoma in situ and with a poor clinical prognosis ${ }^{57,90}$ (FIC. 3). Aberrant expression of MMPs and HIFl $\alpha$ and their effect on HNSCC progression are discussed later.

Among signalling pathways that commonly drive tumour development, the PI3K-AKT-mTOR pathway is the most frequently altered oncogenic pathway in HNSCC $^{59,91}$. Components of this pathway are genetically altered in most HNSCC tumours, with frequent alterations in PIK3CA occurring by both mutation (14\%) and gene amplification (16\%). Loss of function of phosphatase and tensin homologue (PTEN), a negative regulator of $\mathrm{PI} 3 \mathrm{~K}-\mathrm{AKT}$ signalling, results from both genetic and epigenetic alterations and occurs in $~ 30 \%$ of HNSCC tumours ${ }^{92}$ (FIG. 3). STAT3 signalling is hyperactivated in HNSCC and correlates with poor prognosis, although STAT3 is rarely mutated ${ }^{93,94}$. Mutations in the genes encoding the protein tyrosine phosphatase receptors (PTPRs) PTPRT and PTPRD, which occur frequently in HNSCC, have been demonstrated as one cause of STAT3 hyperactivation in head and neck cancers ${ }^{95,96}$. STAT3 signalling drives the expression of genes that promote cellular proliferation and survival as well as genes encoding growth factors and cytokines that promote immunosuppression (such as VEGF, IL-6, IL-10 and TGF $\beta)^{94}$. Oncogenic signalling by the WNT- $\beta$-catenin pathway also contributes to HNSCC ${ }^{97}$. Last, the RAS-MAPK pathway, which contributes to the growth and survival of HNSCC tumour cells, was found by one group to be infrequently mutated in HNSCC tumours, whereas this pathway was found by another group to be mutated in $18 \%$ of HNSCC tumours ${ }^{59}$.

\section{Tumour microenvironment}

The tumour microenvironment (TME) in HNSCC is a complex and heterogeneous mix of tumour cells and stromal cells, which include endothelial cells, cancerassociated fibroblasts (CAFs) and immune cells. Tumour cells and CAFs produce growth factors, such as VEGF, which recruit endothelial cells, stimulating neovascularization and supply of oxygen and nutrients to the tumour. In turn, endothelial cells secrete factors that support the survival and self-renewal of CSCs ${ }^{43}$. CAFs have a key role in HNSCC progression and are distinguished from normal fibroblasts by a persistent state of activation and expression of $\alpha$-smooth muscle actin ( $\alpha \mathrm{SMA})^{98}$. CAFs secrete a broad range of growth factors (such as EGF, VEGF and HGF), cytokines (such as IL-6) and chemokines that promote tumour cell growth, angiogenesis and recruitment of immunosuppressive immune cells ${ }^{99,100}$. In addition, CAFs are the primary source in the TME of MMPs ${ }^{99,100}$, which are involved in degradation and remodelling of the extracellular matrix (ECM) and the release and activation of matrix-embedded growth factors (such as FGFs, VEGF and TGF $\beta$ ) that further stimulate tumour cell proliferation, angiogenesis and immunosuppression. Elevated aSMA levels in HNSCC tumours correlate with poor prognosis ${ }^{101}$.

The TME of HNSCC tumours also includes innervating neurons derived from the peripheral nervous system. In addition, HNSCC tumours contain newly formed adrenergic neurons whose presence acts to stimulate tumour growth ${ }^{102}$. Loss of TP53 seems to have a role in reprogramming sensory neurons in the HNSCC TME to an adrenergic, tumour-promoting phenotype ${ }^{102}$.

Immune evasion. The immune component of the HNSCC TME consists of tumour-infiltrating lymphocytes (TILs; including T cells, B cells and natural killer (NK) cells) and myeloid lineage cells (including macrophages, neutrophils, dendritic cells and myeloid-derived suppressor cells (MDSCs)). In general, HNSCC tumours are highly infiltrated by immune cells, although the extent and composition of the immune cell infiltrate varies according to anatomical subsite and aetiological agent (smoking versus HPV) ${ }^{103,104}$. Furthermore, distinct immune phenotypes have been identified for HNSCC tumours, and several molecular signatures, incorporating diverse markers, have been identified as classifiers ${ }^{105-107}$. These signatures might prove useful for predicting response to different therapies, particularly checkpoint inhibition. High levels of TILs generally correspond to better outcomes in HNSCC but this is dependent on the balance of cells with anti-tumour activity (effector $\mathrm{T}\left(\mathrm{T}_{\text {eff }}\right)$ cells) versus those with immunosuppressive activity (regulatory $\mathrm{T}$ $\left(\mathrm{T}_{\text {reg }}\right)$ cells) in the TIL population ${ }^{108,109}$. Numerous studies have shown that the TME of most HNSCC tumours is highly immunosuppressive $\mathrm{e}^{104,110}$. Antitumour immunity in the TME is mediated largely by $\mathrm{T}_{\text {eff }}$ cells and $\mathrm{NK}$ cells, whereas immune suppression and tumour cell growth is mediated by $\mathrm{T}_{\text {reg }}$ cells, MDSCs and M2 macrophages. Elevated levels of $\mathrm{CD}^{+} \mathrm{T}_{\text {eff }}$ cells and NK cells in the TME are associated with improved survival ${ }^{100}$. By contrast, elevated levels of $\mathrm{T}_{\text {reg }}$ cells, MDSCs, neutrophils or M2 macrophages are associated with advanced-stage HNSCC or a poor prognosis ${ }^{100}$.

The immune cell abundance and composition of HPV-positive tumours and HPV-negative tumours are notably different ${ }^{103,104}$. HPV-positive tumours typically have a greater abundance of TILs than HPV-negative tumours. Importantly, patients with HPV-positive tumours containing high levels of TILs have excellent outcomes, whereas patients with HPV-positive tumours containing low levels of TILs exhibit survival outcomes similar to those of patients with HPV-negative HNSCC ${ }^{111}$.

HNSCC tumours evade immune surveillance by a number of different mechanisms. The milieu of the HNSCC TME is rich in immunosuppressive growth factors and cytokines that promote recruitment or activity of MDSCs, $\mathrm{T}_{\text {reg }}$ cells and M2 macrophages while inhibiting the anti-tumour effects of $\mathrm{T}_{\text {eff }}$ cells and NK cells, of which IL-6, IL-10, VEGF and TGF $\beta$ are particularly important ${ }^{99}$. Factors in the HNSCC TME, including IL-10 and TGF $\beta$, also promote macrophage polarization to the immunosuppressive M2 phenotype ${ }^{112}$. Genetic and epigenetic alterations result in decreased tumour 
cell levels of human leukocyte antigen (HLA) and defects in antigen processing, leading to decreased recognition and cytolysis of tumour cells ${ }^{113,114}$. In addition, HNSCC tumours, particularly advanced-stage cancers, demonstrate upregulation of PDL1, which attenuates the cytolytic activity of T cells ${ }^{7,8}$. Similarly, MDSCs and $\mathrm{T}_{\text {reg }}$ cells recruited to the HNSCC TME express PDL1 and another immunosuppressive molecule, cytotoxic T lymphocyte antigen 4 (CTLA4), respectively. In the specific case of HPV-positive HNSCC, the viral E5, E6 and $\mathrm{E} 7$ proteins promote immune evasion by effects on cellular gene and protein expression in tumour cells ${ }^{115,116}$. The frequent loss in HPV-positive HNSCC of TRAF3, which encodes a protein that functions in anti-viral immunity, also probably contributes to immune evasion in HPV-positive disease ${ }^{117,118}$.

Hypoxia. HNSCC tumours are also characterized by hypoxia, with roughly similar levels of hypoxia in HPV-positive and HPV-negative tumours of the same stage $^{119}$. High levels of hypoxia in tumours portend a poor prognosis and resistance to radiation therapy ${ }^{120-124}$. Hypoxia induces the expression of HIF1a, a subunit of the transcription factor HIF1, which drives expression of a range of genes encoding proteins that promote angiogenesis (VEGF) and degradation of ECM (MMPs). HIF1 also upregulates tumour cell expression of glucose transporters (for example, GLUT1) and enzymes that contribute to metabolic reprogramming of the tumour from oxidative phosphorylation to glycolysis (known as the Warburg effect ${ }^{73}$. In tumour-infiltrating immune cells, hypoxia and HIF1 induce expression of pro-inflammatory and immune-modulatory cytokines and chemokines (such as IL-1 $\beta$ and TNF) ${ }^{73}$.

The oral microbiota. An emerging field of study is the role of the microbiota in the HNSCC TME, particularly in oral cancer. Poor oral health is associated with oral cancer, as well as other cancers, and tobacco, alcohol and HPV are all known to modulate the composition of the oral cavity microbiota ${ }^{125}$. Specific bacterial and fungal signatures that are enriched in oral cancer have been reported and continue to be refined ${ }^{126,127}$.

\section{Metastasis}

Multiple pathways and processes contribute to the invasion and metastasis of HNSCC tumour cells. MMPs produced by both tumour and stromal cells in the TME have an integral role in degrading and remodelling the ECM, thereby promoting tumour cell invasion. High levels of MMP2, MMP9 and MMP13 in HNSCC tumours are associated with invasion, metastasis and poor prognosis ${ }^{128-130}$. Interestingly, the HNSCC CSC marker CD44 serves as a cell surface receptor that binds to and promotes the activity of MMP9 (REFS ${ }^{44,131}$ ). CD44 and MMP9 colocalize to the invasive front of HNSCC tumours and their expression levels correlate with metastasis ${ }^{132}$.

The epithelial-mesenchymal transition (EMT; the conversion of tumour cells from an epithelial to a mesenchymal phenotype) has a key role in HNSCC metastasis. Cells that undergo EMT exhibit downregulation of E-cadherin, upregulation of vimentin, reduction of cell adhesion and enhanced migration and invasiveness. EMT-associated changes in E-cadherin and vimentin levels are associated with increased metastasis of HNSCC tumours ${ }^{100,133}$. The transcription factors TWIST, SNAIL and SLUG mediate downregulation of E-cadherin during EMT, with co-expression of TWIST, SNAIL and HIF1 a correlating with high rates of HNSCC metastasis $^{134,135}$. Hypoxic conditions in the TME can drive EMT in tumour cells, as HIFla induces the expression of vimentin, TWIST and SNAIL ${ }^{100}$; this mechanism may explain the association between elevated levels of hypoxia and HNSCC metastasis ${ }^{120,121,134}$. The process of EMT is also closely linked with the acquisition of stem cell properties, including expression of HNSCC CSC markers $^{135}$. Tumour cell expression of CD44, CD133 or ALDH1 is closely associated with metastasis in HNSCC $^{45}$. Similarly, OCT3, OCT4 and NANOG promote invasiveness of HNSCC cells and their presence may serve as a predictor of metastasis ${ }^{45}$.

Of note, EMT is not a fixed and irreversible process ${ }^{136}$ but instead can be context-dependent, occurring in distinct cellular populations at particular sites within the tumour ${ }^{136,137}$. Reversal of EMT may be necessary for the establishment of macrometastatic tumour sites, underscoring the plasticity of the process ${ }^{136}$. Furthermore, tumour cells may exhibit only partial EMT (p-EMT), a partial acquisition of EMT markers or properties. Single-cell transcriptomic analyses of primary and metastatic HNSCC tumours revealed that cells with p-EMT are localized to the leading edge of tumours and that p-EMT can serve as an independent predictor of metastasis, tumour grade and detrimental pathological features $^{137}$.

The capacity of HNSCC tumour cells to metastasize also requires an ability to detach from the basement membrane and associated ECM components. Typically, when normal epithelial cells detach they lose access to key survival factors and undergo a form of programmed cell death termed anoikis ${ }^{138}$. Metastasis is dependent on suppression of, or acquired resistance to, anoikis. Growth factors and cytokines in the TME, particularly IL-6, EGF and HGF, activate tumour cell signalling pathways, including the RAS-MAPK, PI3K-AKT-mTOR and STAT3 pathways, which suppress anoikis ${ }^{100,139,140}$. Of note, anoikis-suppressive factors in the TME are derived from CAFs, endothelial cells, infiltrating immune cells and the tumour cells themselves ${ }^{100}$, underscoring the complex crosstalk between cell types that contributes to metastasis in HNSCC.

\section{Diagnosis, screening and prevention Clinical presentation}

HNSCC is a cancer of adults, with a median age at diagnosis of 66 years for HPV-negative HNSCC, 53 years for HPV-positive HNSCC and 50 years for EBV-positive HNSCC $^{16,17,141}$. Irrespective of environmental or viral aetiology, the risk of all forms of HNSCC is substantially higher in men than in women. The classic presenting symptoms of HNSCC depend on both the anatomical site of the primary tumour and the aetiology of the tumour (environmental carcinogens, HPV or EBV). 
Oral cavity tumours. Cancers of the oral cavity, including the mobile tongue, floor of the mouth, buccal mucosa, alveolar ridges, retromolar trigone and hard palate, classically present with a non-healing mouth sore or ulcer. Oral cavity tumours are often diagnosed at an early stage owing to the patient's self-identification of the mass lesion and symptoms that interfere with the fundamental functions of eating and speaking, such as pain with chewing or dysarthria (difficulty speaking). Clinical suspicion should be heightened by the coexistence of risk factors for environmental carcinogenesis, including use of smokeless or combustible tobacco, areca nut and betel quid, and alcohol, and poor dentition.

p16 ${ }^{I N K 4 A}$-negative oropharyngeal and hypopharyngeal tumours. Primary tumours of the oropharynx, including the base of the tongue, the palatine tonsils, the soft palate and the hypopharynx, typically become symptomatic at a later stage, owing to their hidden anatomical location. When present, symptoms such as dysphagia (difficulty eating), odynophagia (pain when swallowing) or otalgia (ear pain) often herald a more advanced tumour. A personal history of combustible tobacco and alcohol use should raise the index of suspicion when such non-specific pharyngeal symptoms are present.

Laryngeal tumours. Patients with cancers of the larynx frequently present with voice changes or florid hoarseness, resulting in diagnosis at an early stage. If tumours are neglected, patients can present with dyspnoea (difficulty breathing) and, ultimately, airway obstruction, prompting tracheostomy. The behavioural risk factors of combustible tobacco and alcohol use are multiplicative at the larynx and other HPV-negative sites ${ }^{142}$.

p16 INK4A -positive oropharyngeal tumours. Patients with HPV-related cancers of the oropharynx most commonly present with a new, painless level II (lymph nodes at the upper jugular level) neck mass and an asymptomatic primary tumour. Because the risk factors for HPV-negative HNSCC are often absent, keen awareness of the twenty-first century epidemic of HPV-related HNSCC in North America and Western Europe is crucial for appropriate suspicion and early diagnosis. The dominant risk factors for HPV-related HNSCC include male sex and multiple life-time sexual partners ${ }^{34}$. However, the average latency period of 10-30 years from initial oral infection to ultimate diagnosis of $\mathrm{p} 16^{\mathrm{INK} 4 \mathrm{~A}}$-positive oropharyngeal cancer means that risk factors might be remote ${ }^{143}$. Thus, in all adults, a new neck mass is considered malignant until demonstrated otherwise. In a systematic review of minimally invasive transoral surgery to diagnostically assess an occult primary HNSCC tumour presenting as a cervical neck mass, the majority of tumours were p16 ${ }^{\mathrm{INK} 4 \mathrm{~A}}$-positive ${ }^{144}$. Diagnostic transoral robotic surgery, including palatine and lingual tonsillectomies, identified $\sim 70 \%$ of primary HNSCC tumours, which were most commonly localized in the base of the tongue ${ }^{145}$.

EBV-positive nasopharyngeal tumours. The most common presenting symptoms of nasopharyngeal carcinoma include a cervical neck mass, epistaxis (nosebleed) and unilateral nasal obstruction ${ }^{146}$. Owing to the proximity of the nasopharynx to the base of the skull, patients with more advanced tumours can present with conductive hearing loss or cranial nerve palsies. In east and southeast Asia, where EBV-positive nasopharyngeal carcinoma is endemic, an adult presenting with a new neck mass requires a complete head and neck evaluation with close attention to the nasopharynx.

\section{Diagnosis}

Histopathology. The diagnosis of HNSCC must be established by biopsy of the primary tumour and/or neck mass ${ }^{147}$. The biopsy method depends upon the location of the lesion. Primary tumours are typically approached with cup forceps, incisional biopsy or excisional biopsy, whereas the suspicious cervical neck mass should undergo fine needle aspiration (FNA). Excisional biopsy of a neck mass is not recommended unless the FNA biopsy sample has been persistently non-diagnostic, a primary site has not been identified on cross-sectional imaging or panendoscopy and/or lymphoma is suspected owing to concurrent non-cervical lymphadenopathy.

As HNSCC is derived from the stratified epithelium of the upper aerodigestive mucosa, the histopathological spectrum is characterized by the extent of cellular atypia and squamous differentiation (FIG. 6). A well-differentiated tumour closely resembles the stratified epithelium, with mature-appearing cells organizing into layers with irregular keratinization, most classically manifesting as a 'keratin pearl' (FIG. 6a). A poorly differentiated tumour is characterized by immature cells with nuclear pleomorphism and atypical mitoses, with minimal to no organized stratification or keratinization (FIG. 6b). Of note, the observed extent of squamous differentiation is closely associated with aetiology: HPV-negative HNSCCs are more often moderately or well differentiated, with preservation of stratification and keratinization, whereas HPV-positive HNSCCs are poorly differentiated or even display basaloid morphology on histopathological analysis ${ }^{57}$.

The diagnosis of HNSCC can usually be made on the basis of routine histopathology with haematoxylin and eosin staining. However, in the case of poorly differentiated or basaloid tumours, immunohistochemistry may be necessary to confirm an epithelial origin. HNSCC tumours routinely stain with pancytokeratin antibodies and markers of squamous differentiation, including antibodies to cytokeratin 5, cytokeratin 6 and p63 (REF. ${ }^{148}$ ). HPV testing is mandatory for all oropharyngeal or unknown primary tumours, as it is a major determinant of modern staging and prognosis. Multiple techniques are available for determination of HPV status, including detection of E6 and E7 mRNA (by reverse transcription PCR), HPV DNA (by PCR or in situ hybridization) or the cell cycle protein p16 ${ }^{\mathrm{INK} 4 \mathrm{~A}}$ (by immunohistochemistry) ${ }^{143}$. Consensus recommendations from the College of American Pathologists include testing all newly diagnosed oropharyngeal cancers for HPV by immunohistochemistry for $\mathrm{p} 16^{\mathrm{INK} 4 \mathrm{~A}}$, with a diagnostic threshold of diffuse nuclear and cytoplasmic staining in $>70 \%$ of tumour cells ${ }^{149}$ (FIG. 6c,d). Of note, the level of p16 $6^{\mathrm{INK} 4 \mathrm{~A}}$ 

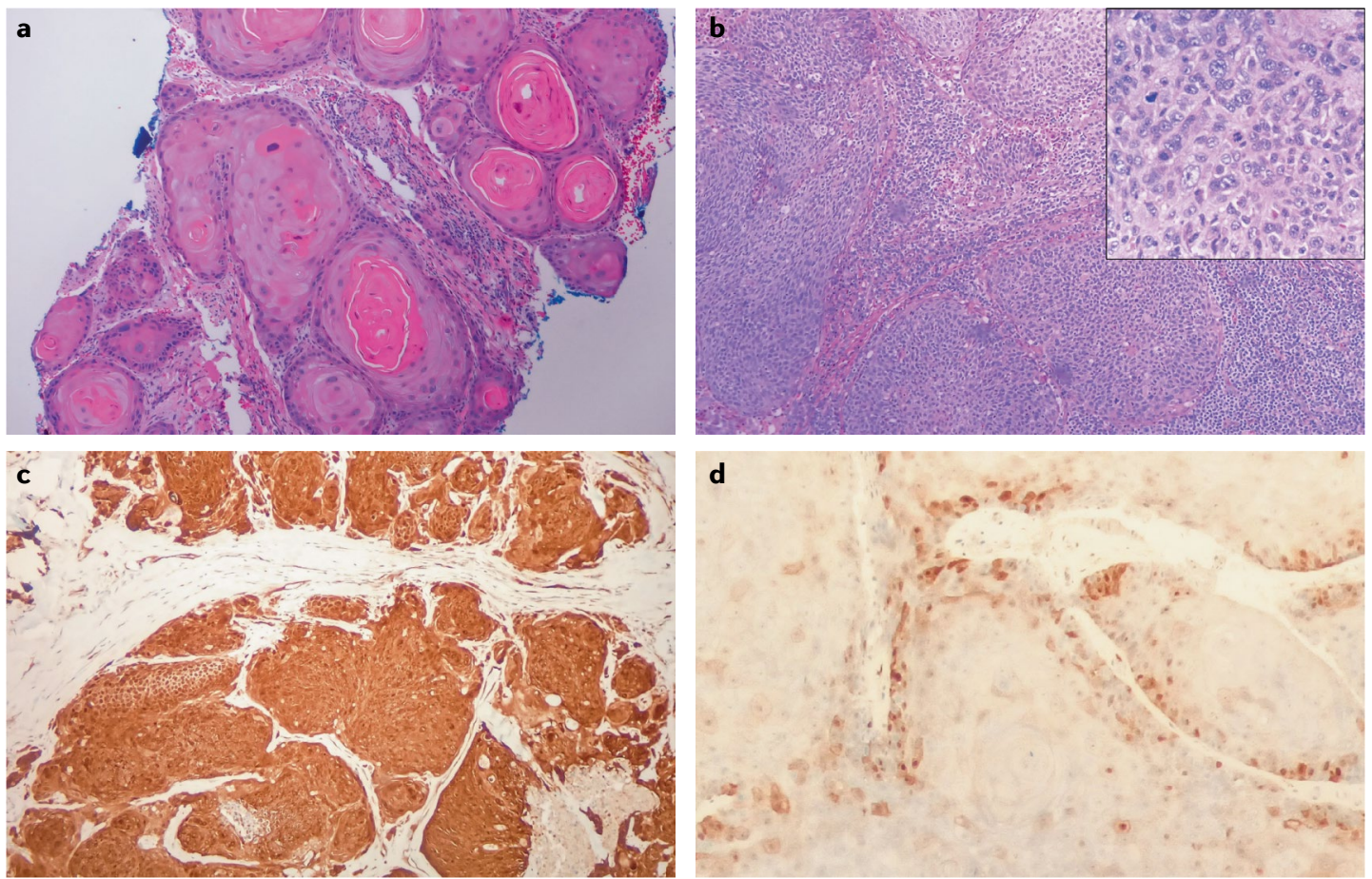

Fig. 6 | Histopathology of HNSCC. a | Well-differentiated squamous cell carcinoma of the oral tongue, demonstrating mature cells with semi-organized keratinization and featuring a 'keratin pearl' $(\times 10)$. $\mathbf{b} \mid$ A poorly differentiated squamous cell carcinoma of the base of the tongue $(\times 10)$. Inset shows immature cells with nuclear pleomorphism and atypical mitoses, without apparent stratification or keratinization $(\times 40)$. c $\mid$ A p $16^{\text {INK4A }}$-positive oropharyngeal squamous cell carcinoma characterized by diffuse nuclear and cytoplasmic staining for the cell cycle protein $\mathrm{p} 16^{\text {INK4A }}$ by immunohistochemistry, indicative of human papillomavirus (HPV)-positive disease $(\times 10)$. d $\mid \mathrm{A}$ p16 $6^{\text {INK4A }}$-negative oropharyngeal squamous cell carcinoma demonstrates minimal staining by the same anti-p $16^{\text {INKAA }}$ antibody, a staining intensity that is indicative of HPV-negative disease $(\times 40)$. In parts $\mathbf{a}$ and $\mathbf{b}$, staining is with haematoxylin and eosin. HNSCC, head and neck squamous cell carcinoma. Images courtesy of G. Tranesh, University of Arizona.

is not a direct measure of HPV infection; $\mathrm{p} 16^{\mathrm{INK} 4 \mathrm{~A}}$ is upregulated on the degradation of $\mathrm{RB}$ and, as such, is a functional surrogate for levels of the oncoprotein E7.

Staging. Stage and HPV status are now recognized as the major determinants of HNSCC prognosis in North America and Western Europe, although HPV-positive HNSCC was not identified as a disease with a distinct aetiology, molecular characteristics and prognosis until $2010\left(\right.$ REF. $\left.^{150}\right)$. Until 2017, the staging of HNSCC involved classification of disease within each classic anatomical subsite (oral cavity, oropharynx, hypopharynx or larynx) according to the tumour-node-metastasis (TNM) system, independent of HPV status ${ }^{151}$. In 2017, the Union for International Cancer Control (UICC) and the American Joint Commission on Cancer (AJCC) released the eighth edition of the Cancer Staging Manual ${ }^{152}$. This edition contains three important changes to improve hazard discrimination and outcome prediction across HNSCC, including the addition of depth of invasion to tumour staging in oral cavity cancers, the addition of extracapsular nodal extension to nodal staging in non-viral HNSCC and codification of a novel staging system for HPV-positive HNSCC ${ }^{152}$. The new staging system for $\mathrm{p} 16^{\mathrm{INK} 4 \mathrm{~A}}$-positive oropharyngeal cancer has improved prognostic discrimination compared with the seventh edition, when applied to retrospective cohorts $^{153-155}$. The revision of nodal staging, in particular the classification of any number of ipsilateral nodes of $<6 \mathrm{~cm}$ (seventh edition, N1-N2b) as N1 disease, and the influence of nodal stage on overall stage group, resulted in a large stage migration from stages III-IVa in the seventh edition to stages I-II in the eighth edition. Although hazard discrimination has probably improved for HPV-positive HNSCC, prospective outcomes data are needed to validate the eighth edition. Of note, the eighth edition does not account for tobacco use, which may be an important modifier of prognosis in $\mathrm{p} 16^{\mathrm{INK} 4 \mathrm{~A}}$-positive oropharyngeal cancer ${ }^{150}$. Unlike HPV-positive HNSCC, current staging of nasopharyngeal carcinoma in the eighth edition is strictly based on anatomy, without incorporation of viral or environmental aetiology. Nomograms incorporating baseline plasma EBV DNA into the staging system significantly improve hazard discrimination for EBV-positive nasopharyngeal carcinoma, which is likely to be incorporated in subsequent editions of the UICC/AJCC Cancer Staging Manual ${ }^{156,157}$.

Following histopathological confirmation of HNSCC, the staging evaluation includes the following procedures in all patients irrespective of HPV status: complete head and neck examination with direct inspection of the oral cavity and fibre-optic nasopharyngolaryngoscopy as indicated; cross-sectional imaging of the head and neck by CT or MRI to establish the extent of locoregional disease; and chest CT to rule out distant metastatic disease. Where available, PET-CT 
is preferred for distant metastatic staging in patients with locally advanced tumours or nodal disease ${ }^{158}$. In patients with tobacco-related HNSCC, the risk of a second tobacco-related primary tumour of the upper aerodigestive tract also warrants staging with panendoscopy under anaesthesia, including direct laryngoscopy, oesophagoscopy and bronchoscopy ${ }^{159}$. As patients with HPV-positive or EBV-positive HNSCC rarely manifest SPTs, panendoscopy can be omitted in most cases ${ }^{143}$.

\section{Primary prevention}

'Primary prevention' involves interventions to reduce the incidence of disease in the first place, by decreasing exposures, altering modifiable behaviours or increasing resistance in healthy people who are at risk. When considered as a target for primary prevention, most cases of HNSCC would indeed be preventable with successful global elimination of tobacco use and implementation of HPV vaccination.

Tobacco and areca nut use. Worldwide, the majority of HNSCC cases are caused by individual use of tobacco, a known carcinogen. Tobacco use, whether smokeless (chew or snuff) or combustible (cigarettes, cigars or pipes), and chewing of areca nut products including betel quid, are modifiable risk factors. Evidence-based interventions for tobacco cessation at the individual level include programmes of behavioural and psychological support that identify tobacco users, provide personalized advice to quit, assess readiness to quit, provide assistance with a quit attempt and arrange follow-up. Quit attempts are more likely to be successful with the use of at least one of seven FDA-approved first-line pharmacotherapies, including five methods of nicotine replacement therapy (the nicotine patch, gum, lozenge, nasal spray or oral inhaler) and two non-nicotine oral medications, bupropion and varenicline ${ }^{160}$. Evidence-based interventions at the national policy level have been ranked according to cost-effectiveness by the WHO, which classifies the following four strategies as 'best buys': increased excise taxes on tobacco products; large graphic warnings on cigarette packages; comprehensive bans on tobacco advertising; and enforcement of a national, comprehensive, ban on smoking in public spaces ${ }^{161}$. Unlike tobacco use, for which the WHO has published evidence-based policies for cessation, no global policy exists for the control of areca nut or betel quid use ${ }^{162}$. As with tobacco, the areca nut is addictive, although the biological basis for addiction is less well understood. Research into both the mechanisms and social determinants of dependence is required in order to advance prevention and cessation programmes.

HPV vaccination. Three prophylactic $H P V$ vaccinations are currently approved by the FDA and each is recommended for a specific sex and age group: the bivalent vaccine (HPV-16 and HPV-18) for females aged 9-26 years, the quadrivalent vaccine (HPV-16, HPV-18, HPV-6 and HPV-11) for females and males aged 9-26 years, and the nonavalent vaccine (HPV-16, HPV-18, HPV-6, HPV-11, HPV-31, HPV-33, HPV-45, HPV-52 and HPV-58) for females and males aged 9-45 years. Whereas the indications for prophylactic HPV vaccination were established from the results of randomized, placebo-controlled trials evaluating the burden of anogenital HPV infections, the effectiveness against oral HPV infection has been analysed retrospectively in three populations. In Costa Rica, a phase III trial of the bivalent vaccine demonstrated $93 \%$ efficacy against oral infection with HPV-16 or HPV-18 (REF. ${ }^{163}$ ). Young adults aged 18-30 years with a self-reported history of HPV vaccination demonstrated a considerably lower rate of vaccine-type-specific oral HPV infection, in repeated cross-sectional data analysis of the National Health and Nutrition Examination Survey (NHANES) 2009-2014 (REF. ${ }^{164}$ ). Finally, a cross-sectional study of NHANES participants aged 18-33 years estimated the individual and population level efficacy of the quadrivalent HPV vaccine. Efficacy against HPV vaccine-type-specific infection was $88 \%$ for oral HPV; however, the population-level effect on the burden of oral HPV-16, HPV-18, HPV-6 and HPV-11 infections was only $17 \%$ overall, $25 \%$ in women and $7 \%$ in men, owing to low vaccination rates ${ }^{165}$.

The US Centers for Disease Control and Prevention (CDC) Advisory Committee on Immunization Practices recommends $\mathrm{HPV}$ vaccination for all children and adults aged 9-26 years ${ }^{166}$. More recently, the FDA approved HPV vaccination for adults aged 27-45 years who had not been adequately vaccinated earlier ${ }^{167}$. Currently, two doses of the vaccine are recommended for children aged 15 years or younger, whereas three doses are recommended for those older than 15 years. Although widespread vaccination has the potential to eliminate HPV-positive HNSCC, the low vaccination rates and exponential increase in incidence observed among men in the USA indicate that herd immunity is at least a generation away ${ }^{34}$.

\section{Secondary prevention}

Secondary prevention refers to the early detection of latent, asymptomatic disease and subsequent interventions to halt disease progression to a harmful state. In cancer, secondary prevention typically involves screening, such as mammography to find and treat early stage breast cancers or Papanicolaou smears to identify and ablate pre-cancerous HPV lesions of the cervix. Although recognized OPLs, including leukoplakia, erythroplakia and dysplastic leukoplakia, are associated with an increased risk of HPV-negative HNSCC, most OPLs themselves do not transform into invasive cancer ${ }^{168,169}$. Rather, HPV-negative HNSCC arises from the grossly normal upper aerodigestive mucosa subject to 'epithelial field cancerization', where chronic exposure to carcinogens has resulted in molecular changes preconditioning towards malignant progression ${ }^{50}$. As such, patients with a first HPV-negative HNSCC are at high risk of a SPT of the upper aerodigestive tract, including in the head and neck, oesophagus or lung ${ }^{159}$. Due to the alarming rate of SPT formation, HNSCC has been a fertile field for the evaluation and eventual failure of multiple chemoprevention agents. For example, in the 1980s, high-dose isotretinoin, a synthetic vitamin A analogue, was found to reverse OPLs and prevent formation of SPTs in two landmark randomized trials; however, ultimately, high-dose isotretinoin was too toxic for chronic daily use 
and has been abandoned ${ }^{170,171}$. More recently, the EPOC trial investigated erlotinib, a small-molecule inhibitor of EGFR, in patients with OPLs who had loss of heterozygosity $(\mathrm{LOH})$ at $9 \mathrm{p}$ and $3 \mathrm{p}$. Although erlotinib was found to be ineffective, $\mathrm{LOH}$ was prospectively confirmed as a molecular biomarker of oral cancer risk ${ }^{172}$.

The development of an effective and tolerable chemoprevention agent against HPV-negative HNSCC remains an elusive but noble ambition. Modern trials of chemoprevention of tobacco-related cancers now focus on the cytoprotective, anti-inflammatory or immunomodulatory potential of repurposed pharmaceutical and nutraceutical compounds that are known to be well-tolerated during prolonged exposure. Ongoing secondary prevention trials include the evaluation of aspirin and other NSAIDs to reduce cyclooxygenase 2 (COX2) signalling ${ }^{173}$; the study of metformin, an anti-hyperglycaemic biguanide that might block mTOR signalling in transforming epithelial cells ${ }^{174}$; and trials of 'green chemoprevention' agents derived from cruciferous vegetables rich in isothiocyanates, which are thought to induce carcinogen detoxification and, possibly, innate immunity ${ }^{175,176}$.

\section{Screening}

No validated tool exists for screening for HPV-positive HNSCC. HPV vaccinations are effective only for primary prevention, as their primary mechanism involves induction of anti-L1 capsid antibodies, which block the first infection step of viral entry. Furthermore, unlike cervical cancer, HPV-positive HNSCC lacks a cytological or gross precursor lesion. Although epidemiology studies have consistently demonstrated an association between oral HPV DNA detection or anti-HPV-16 E6 seropositivity and risk of oropharyngeal cancer, the rarity of HPV-positive HNSCC reduces the clinical utility of such measures ${ }^{143}$.

Of note, a randomized, prospective screening trial in 20,174 asymptomatic men was conducted in Hong Kong and demonstrated that plasma EBV DNA detection had 97.1\% sensitivity and $98.6 \%$ specificity for the identification of nasopharyngeal carcinoma ${ }^{42}$. Furthermore, nasopharyngeal carcinoma was detected at a significantly earlier stage in the clinical trial population than in a historical cohort, with $71 \%$ versus $20 \%$ stage I-II disease, respectively.

\section{Management}

The treatment approach to every individual patient is guided by anatomical subsite, stage, disease characteristics, functional considerations and patient wishes. Although natural history is different for HPV-related than for tobacco-related HNSCC, and intensive therapy is more difficult in elderly patients, these considerations do not lead to absolute differences in how patients should be managed.

\section{Surgery, radiation and chemotherapy}

The principal modalities of curative therapy for locally or locoregionally confined HNSCC are resection, radiation and systemic therapy. Treatment planning should aim for the most highly curative approach, while optimizing preservation of function. In patients with a small primary cancer with no clinical nodal involvement, or involvement of only a single node, cure rates of over $80 \%$ can be achieved with single modality intervention (resection or radiation) ${ }^{177}$. Surgery is commonly used for oral cavity cancers, whereas radiation might be more commonly employed for pharyngeal and laryngeal cancers. For laryngeal cancers, a moderately hypofractionated radiation schedule results in better locoregional control and survival than standard radiation therapy ${ }^{178,179}$. Advances in minimally invasive resection, including transoral robotic or laser resection and larynx-preserving partial laryngectomy, as well as improved reconstructive techniques, have extended the indications for primary surgical management in the hands of highly specialized head and neck surgical oncologists ${ }^{180,181}$. Occult metastases in the draining cervical lymph nodes might be present even in patients with small, invasive primary tumours, and use of elective neck dissection improves survival ${ }^{182}$. In the event of treatment failure after single modality radiation or surgery, salvage with the alternative modality offers a high chance of cure $^{181,183}$. For tumours with more advanced tumour or nodal stage, postoperative radiation or chemoradiation, guided by pathological risk factors, reduces the risk of recurrence and improves survival ${ }^{184,185}$.

Pathological features indicative of increased risk of recurrence include extra-nodal extension, close or involved surgical margins, or perineural invasion; when these are present, administration of high-dose cisplatin chemotherapy concurrently with radiation further improves disease-free survival and impacts survival in the highest risk groups ${ }^{184,185}$. The use of tri-modality therapy (that is, the addition of CRT following surgery) is known to increase the late toxicities of radiation, including chronic dysphagia and aspiration, and might increase the risk of non-cancer-related mortality in survivors ${ }^{186}$. For this reason, it is considered important to accurately predict the extent of disease, including the presence of extra-nodal extension, prior to initiation of definitive therapy, to avoid the need to add CRT after what was initially projected to be single modality surgical therapy, as currently happens in $65 \%$ of node-positive HPV-associated oropharyngeal cancers that are managed primarily with resection ${ }^{187}$. Deep-learning neural networks, other radiomics approaches and functional imaging with PET offer promise in improving preoperative diagnostic accuracy ${ }^{188,189}$. Furthermore, there is a $>50 \%$ likelihood of distant metastases in patients with HPV-positive oropharyngeal cancer and more than five nodes involved - such patients are also poor candidates for initial surgical management, given the potential positive impact of chemotherapy on micrometastases ${ }^{177}$.

Definitive treatment with CRT is recommended as non-surgical treatment for patients with advanced $\mathrm{T}$ stage $(\geq \mathrm{T} 3)$, more than one involved node or a single bulky node, and for function preservation. The standard chemotherapy regimen is single-agent cisplatin given at $100 \mathrm{mg} / \mathrm{m}^{2}$ every 3 weeks ${ }^{190}$, with a 3 -year survival of $37 \%$ in a predominantly HPV-negative population; however, in patients who are poor candidates for high-dose cisplatin owing to pre-existing hearing loss or renal injury, weekly 


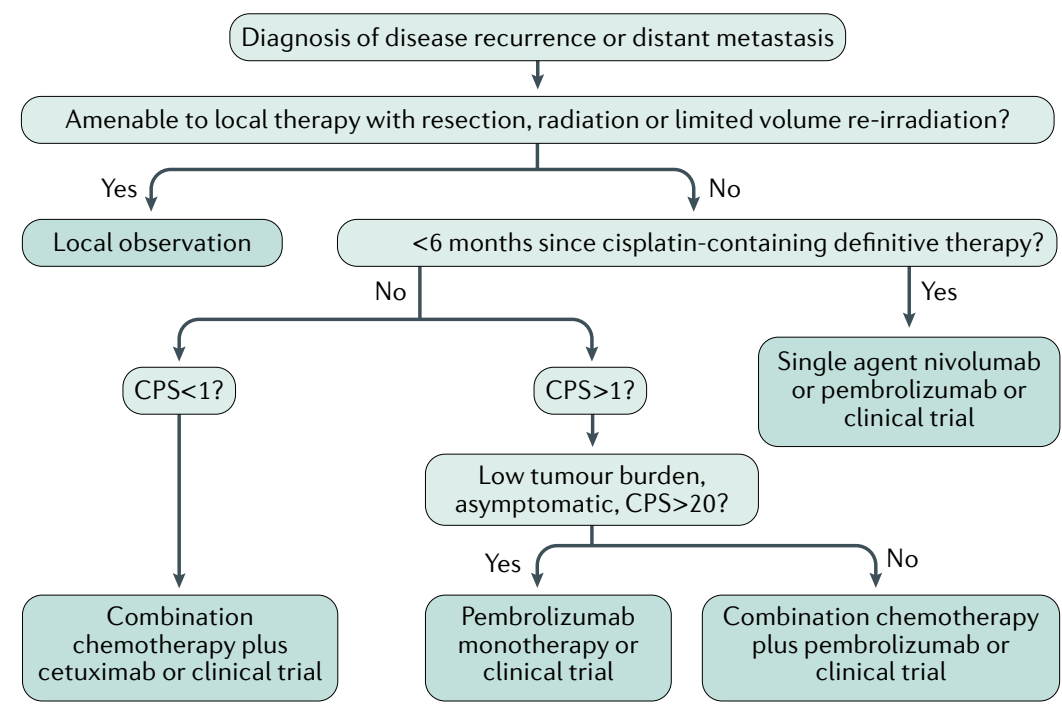

Fig. 7 | Algorithm for treatment decision-making for recurrent and/or metastatic HNSCC. After a diagnosis of recurrent disease or distant metastasis, patients with disease that is amenable to local therapy receive resection, radiation or limited-volume irradiation and are then subject to observation. However, in patients who are not amenable to these therapies, systemic therapy is indicated. Pembrolizumab is chosen in those with PDL1 expression (composite positive score (CPS) $>1$; CPS is (the number of PDL1-staining tumour and immune cells/total number of viable tumour cells counted) $\times 100$ ), given alone in those with high expression and asymptomatic disease (CPS > 20), or given with platinum-based chemotherapy in those with lower expression (CPS 1-19) or higher symptom burden. If PDL1 expression is absent (CPS $<1$ ), platinum doublet chemotherapy with cetuximab is appropriate first-line therapy. Patients who relapse after platinum-based therapy are candidates for nivolumab or pembrolizumab. HNSCC, head and neck squamous cell carcinoma. not to improve OS compared with high-dose cisplatin and radiation ${ }^{197,198}$. However, the proportion of patients showing an objective response is high ${ }^{199}$, and induction chemotherapy can be used to reduce tumour volume in selected situations, such as impending airway obstruction or when a patient is not able to lie flat for radiation simulation but is not a candidate for immediate surgery. Induction chemotherapy may also be used to control disease when curative surgery cannot immediately be accessed, as has been the case in areas with surging incidence of COVID-19 (REF. ${ }^{200}$ ).

The recognition that patients with HPV-associated oropharyngeal cancer, small tumours or a light smoking history enjoy exceptionally high survival ${ }^{150,201}$, but are treated with aggressive multimodality regimens that were evaluated predominantly in the more treatment-resistant HPV-negative population, raises the question as to whether the toxicities and risks of such full-dose therapy are always warranted. A number of single-arm trials seem to demonstrate that in non-smokers with non-bulky tumours, response to induction chemotherapy predicts radiation sensitivity to the extent that radiation dose can be safely reduced from $70 \mathrm{~Gy}$ to $54 \mathrm{~Gy}\left(\mathrm{REFS}^{202,203}\right)$; however, randomized trials are required to confirm that this is an appropriate standard of care.

\section{Immunotherapy}

Among patients with recurrent or metastatic HNSCC, some may be cured by salvage resection, re-irradiation (particularly for nasopharyngeal cancer) ${ }^{204}$ or metastasectomy (particularly for HPV-positive cancer) ${ }^{205}$ (FIG. 7). The remaining patients are considered for systemic therapy. First-line treatment should include the immune checkpoint inhibitor pembrolizumab, an IgG4 humanized antibody to PD1, in patients with PDL1-expressing tumours or microsatellite instability, unless there is a contraindication to immunotherapy because of an underlying autoimmune disorder. A phase III trial compared pembrolizumab monotherapy or the combination of pembrolizumab with a platinating agent and 5-fluorouracil with the same chemotherapy combined with cetuximab ${ }^{9}$. Chemotherapy plus pembrolizumab improved OS compared with chemotherapy plus cetuximab (13 months versus 10.7 months, respectively; HR $0.77, P=0.0034)$, with comparable response and toxicity results. Pembrolizumab alone was non-inferior to combination chemotherapy plus cetuximab among all patients with HNSCC (median OS 11.6 months versus 10.7 months, respectively; HR 0.85). Among patients with expression of the biomarker PDL1, defined as a combined positive score (CPS) of $\geq 20$ or $\geq 1$ (when CPS reflects all PDL1-expressing tumour cells, macrophages and immune cells as a proportion of the total number of tumour cells counted), survival was superior for pembrolizumab monotherapy than for chemotherapy plus cetuximab (14.9 versus 12.3 months, respectively). However, the proportion of patients showing response was higher for the chemotherapy combination; thus, many practitioners will favour pembrolizumab and a chemotherapy combination in patients with a higher disease burden and in those who are more symptomatic, 
while reserving pembrolizumab monotherapy for those with lower tumour volume and the highest PDL1 expression. A minority of patients with HNSCC who are treated with immune checkpoint inhibition may experience accelerated disease progression, often termed hyperprogression ${ }^{206}$. Hyperprogression is most likely in those with HPV-negative disease, or with bulky local or regional recurrence, and when immunotherapy is used without chemotherapy. Although hyperprogression is associated with a worse outcome, objective and even durable responses to chemotherapy can be observed in this setting, and vigilance and rapid alteration in therapy are important to maximize disease control ${ }^{207}$. For patients who experience immune-related adverse events, such as pneumonitis, colitis or other organ injury, treatment interruption and systemic corticosteroids are indicated ${ }^{208}$.

Patients who are not candidates for first-line immunotherapy should receive cetuximab plus combination chemotherapy with a platinating agent and either 5-fluorouracil ${ }^{209}$ or paclitaxel. In the second line, where the prognosis for survival is worse, PD1 inhibition may be reconsidered in some patients despite a history of autoimmune disease, as immune checkpoint inhibitors are also active in patients with cisplatin-refractory disease. Two randomized trials compared PD1 inhibition with either nivolumab ${ }^{7}$ or pembrolizumab ${ }^{210}$ with single-agent standard-of-care chemotherapy in patients whose disease was cisplatin-refractory because they

\section{Box $1 \mid$ Patient experience}

A 58-year-old male patient who is a board member of a large international company underwent platinum-based chemoradiotherapy (70 Gy) for a T1N1 human papillomavirus (HPV)-positive oropharyngeal carcinoma from the left tonsil 1 year ago and describes his experience.

"The reaction from my team at work was superb. We agreed I would focus $100 \%$ on myself, and within a week I was entirely out of the loop. It was sad as well, to find out how quickly you become dispensable. These feelings stayed with me for months, even after I returned to work 6 months later. Disconcerting was the discovery that my treatment for a stage 1 cancer would be basically the same as for advanced cancer.

The first round of chemo went surprisingly well. The radiotherapy sessions were well organized and pleasantly on time. The second chemo session hammered me, and the radiotherapy sessions started taking their toll. My appetite went down as my mouth went dry and my sense of taste slipped away. By the time of the third chemo session I absolutely knew and felt that I was in a war of attrition. Somewhere along the line I developed a persistent diarrhoea that added to the feeling of being drained. Entering my last week of radiotherapy, I was dehydrated, underfed, and weak to the point of having to lie down while waiting for the radiotherapy sessions.

Thankfully some of my worst fears did not materialize, such as being put on a feeding tube or getting radiotherapy burns that would have required additional interventions. During the treatment my throat did swell and became constrained, but in my view this was not the major cause of the relatively mild swallowing or speech problems l experienced. The main factors were having a dry mouth and being foggy-brained. The fogginess took about two months to clear, about as long as it took me to stop carrying a water bottle everywhere all the time. I had anticipated this would take longer and I think it's a benefit of having been irradiated on one side of the throat only.

There are five remaining physical symptoms from my treatment: mouth dryness (back at $85 \%$ of where I was), loss of taste, alopecia spots in my beard and frequent tinnitus in the ears, and lower energy levels. At work and at home and socially things are basically back to normal as well. Generally, I take things easier and I do not stress about the discomforts; it's way better than where I was and could have ended up.

Whatever happens, it's clear that the fear of falling badly sick again and spending days in hospital is something that consciously or unconsciously besets me and probably all surviving patients with cancer." had progressed on cisplatin-based therapy for recurrent and/or metastatic disease, or because cancer had recurred within 6 months of concurrent cisplatin and radiation treatment. In each case, PD1 inhibition improved survival compared with chemotherapy; a small proportion of patients experience very durable benefit and some may benefit from continued therapy after progression $^{211,212}$. For patients with metastatic and/or recurrent HNSCC, many clinical trials are currently available that are exploring novel immunotherapies, as well as the very promising combinations of immune checkpoint inhibition with multi-targeted kinase inhibitors and other anti-angiogenic agents $\mathrm{s}^{213}$.

Throughout treatment, attention to symptom management, functional rehabilitation and appropriate incorporation of palliative care services are key to maintaining performance status and QOL, and supporting patients at the end of life.

\section{Quality of life \\ Patient health and supportive care}

Given the complex nature of everyday functions within the head and neck area, the inherent consequences of HNSCC and its treatment and the increasing choices of treatments have a large effect on the health-related QOL (HRQOL) of patients with HNSCC.

The wide array and combinations of treatments all have their specific sequelae, including physical, emotional, functional and social sequelae, and occupational dysfunction, as well as a profound effect on the families of patients with HNSCC ${ }^{214}$. Furthermore, HRQOL is significantly associated with survival ${ }^{215,216}$. For example, a clinically meaningful association exists between HRQOL scores measured at diagnosis and OS of patients after treatment (HR 0.90, CI 0.86-0.94, $P<0.001)^{217}$. Depending on the primary tumour site, patients with HNSCC might be confronted with specific symptoms, such as oral dysfunction and swallowing and speech problems, during treatment, which often improve 6 months after treatment ${ }^{218}$. However, long-term reduction in QOL in HNSCC survivors (at 10 years) is common $^{219}$. On average, overall HRQOL deteriorated by $11 \%$ when compared with pretreatment, and by $15 \%$ when compared with years 1 and 2 after treatment ${ }^{219}$.

To provide individualized (supportive) care, monitoring HRQOL in a structured manner in clinical research and practice is important. HRQOL is typically assessed using patient-reported outcome measures (PROMs) ${ }^{220}$. Widely used PROMs among patients with HNSCC include the European Organization for Research and Treatment of Cancer (EORTC) Quality of Life Questionnaires (QLQ-C30, QLQ-HN35 and QLQ-HN43) ${ }^{221}$ and the Functional Assessment of Cancer Therapy - Head \& Neck Cancer (FACT-H\&N) ${ }^{222}$.

\section{Survivorship}

With the increase in incidence and improved survival, more people have to cope with living beyond a diagnosis of HNSCC and its treatment (see BOX 1). From a more individualized perspective, this post-diagnosis survival has been defined as "living with, through and beyond a cancer diagnosis" ${ }^{\prime 223,224}$. For HNSCC specifically, patients 
are confronted with numerous, profound disabilities owing to the anatomical complexity of the head and neck region, which can also affect patients' families ${ }^{225}$. Swallowing and speech impairments occur in $~ 50 \%$ of HNSCC survivors following radiotherapy treatment and are often present long term ${ }^{22}$. For example, a majority (68\%) of HNSCC survivors reported voice problems even 10 years after radiotherapy ${ }^{227}$. Furthermore, the 2 -year prevalence of dysphagia is $45 \%$ among HNSCC survivors (all therapies) and is four to eight times more likely to occur in these individuals than in those who have never had cancer ${ }^{228}$.

\section{Moving forwards}

An integrated approach to survivorship care in HNSCC survivors, including (early) rehabilitation, psychosocial care, lifestyle interventions and self-management, are likely to be implemented in the next 5-10 years ${ }^{229}$. In addition, peer support can be important in addressing the general and specific survivorship needs of individual patients ${ }^{230}$.

Substantial efforts are being made to improve HRQOL in patients with HNSCC by developing e-health applications, including self-monitoring of HRQOL and self-help interventions ${ }^{231}$. These innovations carry the promise of sustainably improving supportive care and empowerment of HNSCC survivors ${ }^{232}$. Measurement precision of HRQOL by PROMs can benefit from other data sources as biomarkers and from objective measures (collected using, for example, wearable devices, such as a smart watch, that record and analyse physical activity) ${ }^{233}$. Furthermore, emerging data-driven analytics can be used to study and improve forecasts of HRQOL ${ }^{233}$.

One of the current major challenges is to make collected HRQOL data universally available to patients and clinicians in a meaningful way that will facilitate the design of treatment regimens and post-therapy decisions and indicate appropriate supportive care for the individual patient.

\section{Outlook}

Advances in biotechnology, drug development, robotic surgery, radiotherapy approaches and molecular characterization of human cancers (including HNSCC) in the twenty-first century were expected to lead to improved outcomes for patients with HNSCC. However, despite these advances, outcomes have remained mostly unchanged for the past few decades, especially for HPV-negative HNSCC, and short-term and long-term treatment-associated morbidities are still substantial. Most patients still present with advanced-stage disease and are treated with platinum-based chemotherapy regimens that were approved by the FDA in 1978. Patients who survive their first HNSCC (especially HPV-negative HNSCC) remain at risk for development of a SPT of the upper aerodigestive tract for the rest of their lives. The requirement for multidisciplinary care (including, amongst others, head and neck surgery, radiation oncology, medical oncology, head and neck pathology, speech language pathology, nutrition, prosthodontics and oral medicine) coupled with the rarity of HNSCC in most countries, underscores our recommendation that all patients with HNSCC should be treated in centres with high case numbers and by experienced multidisciplinary teams. An analysis conducted in the USA employing the National Cancer Database showed that patients undergoing curative radiotherapy at facilities with high case numbers and academic centres showed higher survival than patients treated at centres with low case numbers ${ }^{234}$. Even when patients with HNSCC are treated in a multi-institutional chemoradiation clinical trial, the OS of those patients treated at centres that enrol more patients in trials in the USA was significantly longer than that of patients treated at institutions that enrol fewer patients ${ }^{235}$. In a pooled analysis of six randomized, controlled trials in Italy, patients with HNSCC treated at centres with high case numbers showed longer survival than patients treated at centres with low case numbers ${ }^{236}$. Despite improvements in treatment options, particularly the incorporation of immune checkpoint inhibitors into the standard of care, we also recommend that all patients with advanced, incurable HNSCC be offered the opportunity to participate in clinical trials that are based on a strong biological rationale. TABLE 1 lists trials of HNSCC treatments in the National Clinical Trials Network that are currently open for patient enrolment. Fortunately, numerous clinical trials are ongoing and precision medicine approaches are emerging.

\section{HPV-positive HNSCC}

The awareness that HPV contributes to oropharyngeal HNSCC in the majority of patients has markedly affected clinical practice. HPV vaccination has been shown to prevent infection with HNSCC-causing HPV types, including HPV-16 and HPV-18, which are responsible for the majority of HPV-positive HNSCC. In the USA, HPV vaccination is generally administered at the discretion of the paediatrician and the parents, with only Virginia, Rhode Island and the District of Columbia requiring the HPV vaccine for public school attendance. The WHO recommends HPV vaccination as a part of routine vaccinations in all countries. Barriers to widespread vaccination of all girls and boys prior to HPV infection include cost, general vaccine scepticism and concerns that administration of a vaccine against a sexually transmitted virus will encourage sexual activity. Stated simply, HPV vaccination of all children and young adults prior to HPV infection is likely to dramatically reduce, if not eliminate completely, HPV-positive HNSCC. Whereas the incidence of smoking-related HNSCC continues to decline worldwide, that of HPV-positive HNSCC is on the rise ${ }^{237}$. The number of men with HPV-positive HNSCC has now surpassed the number of women with HPV-associated cervical cancer in the USA ${ }^{238}$, suggesting that without widespread HPV vaccination, HPV-positive HNSCC could reach epidemic proportions. We encourage clinicians who treat patients with HNSCC to partner with paediatricians, public health officials and politicians to increase HPV vaccination rates worldwide.

As the pathogenesis of HPV-positive HNSCC seems to be distinct from that of HPV-negative HNSCC, a tantalizing possibility is that HPV-positive tumours 
have unique vulnerabilities that could be exploited for therapeutic purposes. Non-smokers who develop HPV-positive HNSCC generally survive their head and neck cancer, and clinical strategies to date have focused on identifying treatments that are associated with reduced morbidity. The known toxicities of the EGFR inhibitor cetuximab are notably less severe than those of platinum chemotherapy. In an effort to reduce treatment morbidity and maintain the high survival in patients with HPV-positive HNSCC, phase III clinical trials have compared cetuximab plus radiation with cisplatin plus radiation. These studies have demonstrated that cetuximab is inferior to cisplatin in HPV-positive disease, thus precluding this FDA-approved molecular targeting agent as a treatment option for HPV-positive HNSCC $^{5,6}$. Studies leading to the incorporation of immune checkpoint inhibitors, including pembrolizumab and nivolumab, into standard of care for HNSCC have included both patients with HPV-positive and patients with HPV-negative disease. FDA approval of these agents for HNSCC treatment confirmed the power of harnessing the immune system for HNSCC therapy. However, given the improved outcome in most patients with HPV-positive HNSCC, they are less likely to receive these immune checkpoint inhibitors as part of standard of care than patients with HPV-negative HNSCC. Ongoing and future research aimed at elucidating the immune profile of HPV-positive HNSCC is expected to help define therapeutic targets in this population. Mitigating the toxicities associated with standard of care, including platinum chemotherapy and radiation, for these patients remains an important objective.

\section{Molecular characterization of HNSCC}

Substantial progress has been made over the past decade in the molecular characterization of both HPV-positive and HPV-negative HNSCC, culminating in TCGA ${ }^{59,71,72}$. These collective efforts have confirmed the preponderance of TP53 mutations, identified previously unknown NOTCH1 mutations and provided a platform to test precision medicine approaches in HNSCC. Success stories include the recognition that HRAS mutations may serve as predictive biomarkers for treatment with farnesyl transferase inhibitors, such as tipifarnib ${ }^{239}$, and evidence that oncogenic PIK3CA mutations are common and could be used to identify patients with HNSCC who are most likely to benefit from PI3K pathway inhibitors ${ }^{69,240}$. Although some centres are routinely evaluating the mutational profile of HNSCC tumours, the tumours in the majority of patients with HNSCC remain uncharacterized, even when these patients present with recurrent or metastatic disease. Furthermore, the role of tumour mutational burden as a predictive biomarker for immunotherapy remains incompletely understood and efforts are underway to establish guidelines that are designed to harmonize tumour mutational burden evaluation and reporting ${ }^{241}$. Prospective, targeted sequencing, supported by efforts such as the American Association for Cancer Research Project Genie, aim to provide a publicly accessible international cancer registry of real-world data assembled through data sharing ${ }^{242}$. Importantly, single-cell transcriptomic analysis is a powerful new tool for defining diverse intratumoural expression programmes and differences among tumours from different patients, as has been demonstrated in HNSCC ${ }^{137,243}$. In addition,

Table 1 | Current National Clinical Trials Network head and neck cancer trials

\begin{tabular}{|c|c|c|}
\hline $\begin{array}{l}\text { Protocol } \\
\text { number }\end{array}$ & Phase & Protocol \\
\hline EA3132 & $\|$ & $\begin{array}{l}\text { Randomized trial of radiotherapy with or without cisplatin for surgically resected HNSCC with } \\
\text { TP53 sequencing }\end{array}$ \\
\hline EA3161 & II/III & $\begin{array}{l}\text { Randomized trial of maintenance nivolumab versus observation in patients with locally } \\
\text { advanced, intermediate risk HPV-positive OPCA }\end{array}$ \\
\hline EA3163 & II & $\begin{array}{l}\text { Randomized trial of neoadjuvant chemotherapy followed by surgery and postoperative } \\
\text { radiation versus surgery and postoperative radiation for organ preservation of T3 and T4a } \\
\text { NPNSCC }\end{array}$ \\
\hline NRG-HN001 & II/III & $\begin{array}{l}\text { Randomized studies of individualized treatment for nasopharyngeal carcinoma based on } \\
\text { biomarker EBV DNA }\end{array}$ \\
\hline NRG-HN004 & II/III & $\begin{array}{l}\text { Randomized trial of radiotherapy with concurrent MEDI4736 (durvalumab) versus radiotherapy } \\
\text { with concurrent cetuximab in patients with stage III-IVb head and neck cancer with a } \\
\text { contraindication to cisplatin }\end{array}$ \\
\hline NRG-HN005 & II/III & $\begin{array}{l}\text { Randomized trial of de-intensified radiation therapy for patients with early-stage, } \mathrm{p} 16^{+} \text {, } \\
\text { non-smoking-associated oropharyngeal cancer }\end{array}$ \\
\hline RTOG-1008 & ॥ & $\begin{array}{l}\text { Randomized study of adjuvant concurrent radiation and chemotherapy versus radiation alone } \\
\text { in resected high-risk malignant salivary gland tumours }\end{array}$ \\
\hline RTOG-1216 & II/III & $\begin{array}{l}\text { Randomized trial of surgery and postoperative radiation delivered with concurrent cisplatin } \\
\text { versus docetaxel versus docetaxel and cetuximab for high-risk HNSCC }\end{array}$ \\
\hline EAY131 & $\|$ & Molecular analysis for therapy choice (MATCH) \\
\hline S1609 & $\|$ & Dual anti-CTLA4 and anti-PD1 blockade in rare tumours (DART) \\
\hline
\end{tabular}

The table includes trials that were open for patient enrolment as of 15 April 2020. CTLA4, cytotoxic T lymphocyte antigen 4; EBV, Epstein-Barr virus; HNSCC, head and neck squamous cell carcinoma; HPV, human papillomavirus; NPNSCC, nasal and paranasal sinus squamous cell carcinoma; OPCA, oral and pharyngeal cancer. 
increased use of relevant preclinical models, including patient-derived xenografts ${ }^{24,245}$ and organoids ${ }^{246}$, should facilitate the translation of laboratory discoveries to patients with HNSCC.

\section{Immunotherapy}

The importance of the immune system in the development and treatment of HNSCC has long been recognized, leading to early efforts to treat this malignancy using strategies to activate endogenous immunity. The FDA approval of immune checkpoint inhibitors in 2016 for the treatment of recurrent or metastatic disease, and for front-line treatment of inoperable or metastatic cancer in 2019, definitively altered the landscape of HNSCC therapy and clinical trial opportunities. In patients who benefit from these agents, responses seem to be durable ${ }^{7-9}$. However, to date, only a subset of patients with HNSCC are expected to respond to immune checkpoint inhibitors, and reliable predictive biomarkers are needed. Evidence exists that PDL1 expression levels in the tumour may indicate a likelihood of clinical benefit, which led to the 2019 approval of pembrolizumab as first-line therapy in patients with HNSCC whose tumours have a CPS of $\geq 1$ (REF.').

In addition to FDA-approved and emerging agents that target PD1 or PDL1, the anti-CTLA4 monoclonal antibody ipilimumab is also in active clinical development (>20 trials are in progress) for HNSCC treatment. The non-redundant functions of PD1 and CTLA4 in regulating the adaptive immune system serve as the basis for combining PD1 and CTLA4 inhibitors for cancer therapy, including HNSCC, in ongoing clinical trials. Whereas PD1 and PDL1 inhibitors are generally well tolerated, CTLA4 inhibitors are associated with more toxicity ${ }^{247}$, and it will be important to balance efficacy and toxicity when combining these agents. In addition, although adaptive immune cell therapies (for example, chimeric antigen receptor (CAR) T cells) and therapeutic vaccines are not FDA-approved, they are under active investigation in head and neck cancer and represent a promising new avenue for therapy.

The era of immune-oncology has highlighted the limitations of traditional preclinical HNSCC models, including cell lines and xenografts derived from cell lines and patient tumours. Even though patient-derived xenografts are a more accurate model of the patient tumour than cell lines, the xenograft stroma largely consists of mouse cells and these models are generally grown in immunocompromised mice. The advent of immunocompetent HNSCC models offers the opportunity to evaluate immunotherapies in more realistic animal models of HNSCC prior to initiating clinical testing. For example, a report of a syngeneic carcinogen-induced HNSCC tumour in immunocompetent mice demonstrates the ability of these models to assess responses to anti-CTLA4 antibodies ${ }^{248}$. Given the limitations of preclinical models to study immune perturbations, the development of novel clinical trial platforms in conjunction with immune profiling approaches offer opportunities to test the effect of novel immunotherapies in the clinical setting with minimal risk ${ }^{249}$.

Published online: 26 November 2020
1. Stein, A. P. et al. Prevalence of human papillomavirus in oropharyngeal cancer: a systematic review. Cancer J. 21, 138-146 (2015)

2. Isayeva, T., Li, Y., Maswahu, D. \& Brandwein-Gensler, M. Human papillomavirus in non-oropharyngeal head and neck cancers: a systematic literature review. Head Neck Pathol. 6, S104-S120 (2012).

3. Michaud, D. S. et al. High-risk HPV types and head and neck cancer. Int. J. Cancer 135, 1653-1661 (2014)

4. Bonner, J. A. et al. Radiotherapy plus cetuximab for squamous-cell carcinoma of the head and neck N. Engl. J. Med. 354, 567-578 (2006). This study led to the FDA approval of cetuximab for HNSCC.

5. Gillison, M. L. et al. Radiotherapy plus cetuximab or cisplatin in human papillomavirus-positive oropharyngeal cancer (NRG Oncology RTOG 1016): a randomised, multicentre, non-inferiority trial. Lancet 393, 40-50 (2019).

6. Mehanna, H. et al. Radiotherapy plus cisplatin or cetuximab in low-risk human papillomaviruspositive oropharyngeal cancer (De-ESCALaTE HPV): an open-label randomised controlled phase 3 trial. Lancet 393, 51-60 (2019).

7. Ferris, R. L. et al. Nivolumab for recurrent squamous-cell carcinoma of the head and neck N. Engl. J. Med 375, 1856-1867 (2016). This paper describes the clinical trial that led to FDA approval of nivolumab for HNSCC

8. Seiwert, T. Y. et al. Safety and clinical activity of pembrolizumab for treatment of recurrent or metastatic squamous cell carcinoma of the head and neck (KEYNOTE-012): an open-label, multicentre, phase $1 \mathrm{~b}$ trial. Lancet Oncol. 17, 956-965 (2016).

This paper describes the clinical studies that led to FDA approval of pembrolizumab for HNSCC.

9. Burtness, B. et al. Pembrolizumab alone or with chemotherapy versus cetuximab with chemotherapy for recurrent or metastatic squamous cell carcinoma of the head and neck (KEYNOTE-048): a randomised, open-label, phase 3 study. Lancet 394, 1915-1928 (2019).

This paper describes a phase III trial evaluating effect of checkpoint inhibition alone or in combination with chemotherapy in recurren or metastatic HNSCC.

10. Ferlay, J. et al. Estimating the global cancer incidence and mortality in 2018: GLOBOCAN sources and methods. Int. J. Cancer 144, 1941-1953 (2019)

11. Bray, F. et al. Global cancer statistics 2018: GLOBOCAN estimates of incidence and mortality worldwide for 36 cancers in 185 countries. CA Cancer J Clin 68, 394-424 (2018).

12. Ferlay, J. et al. Global Cancer Observatory: Cancer Today. Lyon, France: International Agency for Research on Cancer (accessed 18 September 2020). IARC https://gco.iarc.fr/today (2018).

13. Hashibe, M. et al. Alcohol drinking in never users of tobacco, cigarette smoking in never drinkers, and the risk of head and neck cancer: pooled analysis in the International Head and Neck Cancer Epidemiology Consortium. J. Natl Cancer Inst. 99, 777-789 (2007)

14. Mehanna, $\mathrm{H}$. et al. Prevalence of human papillomavirus in oropharyngeal and nonoropharyngeal head and neck cancer-systematic review and meta-analysis of trends by time and region. Head Neck 35, 747-755 (2013).

15. Jiang, H. et al. Can public health policies on alcohol and tobacco reduce a cancer epidemic? Australia's experience. BMC Med. 17, 213 (2019).

16. Windon, M. J. et al. Increasing prevalence of human papillomavirus-positive oropharyngeal cancers among older adults. Cancer 124, 2993-2999 (2018).

17. Fung, S. Y., Lam, J. W. \& Chan, K. C. Clinical utility of circulating Epstein-Barr virus DNA analysis for the management of nasopharyngeal carcinoma. Chin. Clin. Oncol. 5, 18 (2016)

18. Pulte, D. \& Brenner, H. Changes in survival in head and neck cancers in the late 20th and early 21 st century: a period analysis. Oncologist 15, 994-1001 (2010).

19. Chaturvedi, A. K. et al. Human papillomavirus and rising oropharyngeal cancer incidence in the United States. J. Clin. Oncol. 29, 4294-4301 (2011).
20. Osazuwa-Peters, N. et al. Suicide risk among cancer survivors: head and neck versus other cancers. Cancer 124, 4072-4079 (2018).

21. International Agency for Research on Cancer. Lis of Classifications by cancer sites with sufficient or limited evidence in humans, Volumes 1 to 127 IARC Monographs On The Identification Of Carcinogenic Hazards To Humans. IARC https:// monographs.iarc.fr/agents-classified-by-the-iarc/ (2020).

22. Blot, W. J. et al. Smoking and drinking in relation to oral and pharyngeal cancer. Cancer Res. 48 3282-3287 (1988)

23. Zhang, L. W. et al. Incidence and mortality trends in oral and oropharyngeal cancers in China, 2005-2013. Cancer Epidemiol. 57, 120-126 (2018).

24. World Health Organization. WHO global report on trends in prevalence of tobacco smoking 2015. WHO https://apps.who.int/iris/handle/10665/156262 (2015).

25. Wong, I. C., Ng, Y. K. \& Lui, V. W. Cancers of the lung, head and neck on the rise: perspectives on the genotoxicity of air pollution. Chin. J. Cancer 33 476-480 (2014).

26. Mishra, A. \& Meherotra, R. Head and neck cancer: global burden and regional trends in India. Asian Pac J. Cancer Prev. 15, 537-550 (2014).

27. Guha, N. et al. Oral health and risk of squamous cell carcinoma of the head and neck and esophagus: results of two multicentric case-control studies. Am. J. Epidemiol. 166, 1159-1173 (2007).

28. Freedman, N. D. et al. Fruit and vegetable intake and head and neck cancer risk in a large United States prospective cohort study. Int. J. Cancer 122 , 2330-2336 (2008)

29. Hennessey, P. T., Westra, W. H. \& Califano, J. A. Human papillomavirus and head and neck squamous cell carcinoma: recent evidence and clinical implications. J. Dent. Res. 88, 300-306 (2009)

30. Tsang, C. M., Lui, V. W. Y., Bruce, J. P., Pugh, T. J. \& Lo, K. W. Translational genomics of nasopharyngeal cancer. Semin. Cancer Biol. 61, 84-100 (2020). 
31. Viens, L. J. et al. Human papillomavirus-associated cancers - United States, 2008-2012. Morb. Mortal. Wkly Rep. 65, 661-666 (2016).

32. Gillison, M. L. et al. Prevalence of oral HPV infection in the United States, 2009-2010. JAMA 307, 693-703 (2012). This paper describes the prevalence of oral HPV infection in the USA

33. Chaturvedi, A. K. et al. NHANES 2009-2012 findings: association of sexual behaviors with higher prevalence of oral oncogenic human papillomavirus infections in US men. Cancer Res. 75, 2468-2477 (2015).

34. Gillison, M. L., Chaturvedi, A. K., Anderson, W. F. $\&$ Fakhry, C. Epidemiology of human papillomavirus positive head and neck squamous cell carcinoma. J. Clin. Oncol. 33, 3235-3242 (2015). This paper describes the epidemiology of the emerging increase in HPV-positive HNSCC.

35. Gillison, M. L., Chaturvedi, A. K. \& Lowy, D. R. HPV prophylactic vaccines and the potential prevention of noncervical cancers in both men and women. Cancer 113, 3036-3046 (2008)

36. Rowhani-Rahbar, A. et al. Antibody responses in oral fluid after administration of prophylactic human papillomavirus vaccines. J. Infect. Dis. 200, 1452-1455 (2009).

37. Velleuer, E. \& Dietrich, R. Fanconi anemia: young patients at high risk for squamous cell carcinoma. Mol. Cell Pediatr. 1, 9 (2014)

38. Fang, M., Huang, W., Mo, D., Zhao, W. \& Huang, RAssociation of five Snps in cytotoxic T-lymphocyte antigen 4 and cancer susceptibility: evidence from 67 studies. Cell Physiol. Biochem. 47, 414-427 (2018).

39. Niu, Y. M. et al. Increased risks between Interleukin-10 gene polymorphisms and haplotype and head and neck cancer: a meta-analysis. Sci. Rep. 5, 17149 (2015).

40. Wang, Y., Yang, H., Duan, G. \& Wang, H. The association of the CYP1A1 Ile462Val polymorphism with head and neck cancer risk: evidence based on a cumulative meta-analysis. Onco Targets Ther. $\mathbf{9}$, 2927-2934 (2016).

41. Cadoni, G. et al. A review of genetic epidemiology of head and neck cancer related to polymorphisms in metabolic genes, cell cycle control and alcohol metabolism. Acta Otorhinolaryngol. Ital. 32, 1-11 (2012).

42. Chan, K. C. A. et al. Analysis of plasma Epstein-Barr virus DNA to screen for nasopharyngeal cancer. N. Engl. J. Med. 377, 513-522 (2017).

43. Krishnamurthy, S. et al. Endothelial cell-initiated signaling promotes the survival and self-renewal of cancer stem cells. Cancer Res. 70, 9969-9978 (2010).

44. Faber, A. et al. CD44 as a stem cell marker in head and neck squamous cell carcinoma. Oncol. Rep. 26 321-326 (2011)

45. Yu, S. S. \& Cirillo, N. The molecular markers of cancer stem cells in head and neck tumors. J. Cell Physiol. 235, 65-73 (2020).

46. Zhang, Q. et al. A subpopulation of CD133(+) cancer stem-like cells characterized in human oral squamous cell carcinoma confer resistance to chemotherapy. Cancer Lett. 289, 151-160 (2010).

47. Chiou, S. H. et al. Positive correlations of Oct-4 and Nanog in oral cancer stem-like cells and high-grade oral squamous cell carcinoma. Clin. Cancer Res. 14 4085-4095 (2008).

48. Leon, X. et al. Second, third, and fourth head and neck tumors. A progressive decrease in survival. Head Neck 34, 1716-1719 (2012).

49. Leon, X. et al. Risk of onset of second neoplasms and successive neoplasms in patients with a head and neck index tumour. Acta Otorrinolaringol. Esp. 71, 9-15 (2020).

50. Slaughter, D. P., Southwick, H. W. \& Smejkal, W. Field cancerization in oral stratified squamous epithelium clinical implications of multicentric origin. Cancer 6 , 963-968 (1953).

51. Ryser, M. D., Lee, W. T., Ready, N. E., Leder, K. Z. \& Foo, J. Quantifying the dynamics of field cancerization in tobacco-related head and neck cancer: a multiscale modeling approach. Cancer Res. 76, 7078-7088 (2016).

52. Sheth, S. H., Johnson, D. E., Kensler, T. W. \& Bauman, J. E. Chemoprevention targets for tobacco-related head and neck cancer: past lessons and future directions. Oral. Oncol. 51, 557-564 (2015)

53. Hecht, S. S. Tobacco smoke carcinogens and lung cancer. J. Natl Cancer Inst. 91, 1194-1210 (1999).

54. Hoffmann, D. \& Hoffmann, I. The changing cigarette 1950-1995. J. Toxicol. Env. Health 50, 307-364 (1997).
55. Warnakulasuriya, S. \& Straif, K. Carcinogenicity of smokeless tobacco: evidence from studies in human \& experimental animals. Indian. J. Med. Res. 148, 681-686 (2018)

56. Talamini, R. et al. Combined effect of tobacco and alcohol on laryngeal cancer risk: a case-control study. Cancer Causes Control. 13, 957-964 (2002).

57. Pai, S. I. \& Westra, W. H. Molecular pathology of head and neck cancer: implications for diagnosis, prognosis, and treatment. Annu. Rev. Pathol. 4 49-70 (2009).

58. Brooks, P J \& Theruvathu, J. A. DNA adducts from acetaldehyde: implications for alcohol-related carcinogenesis. Alcohol 35, 187-193 (2005).

59. Cancer Genome Atlas Network. Comprehensive genomic characterization of head and neck squamous cell carcinomas. Nature 517, 576-582 (2015). This paper provides a comprehensive description of the genomic landscape of human HNSCC tumours.

60. Scheffner, M., Huibregtse, J. M., Vierstra, R. D. \& Howley, P. M. The HPV-16 E6 and E6-AP complex functions as a ubiquitin-protein ligase in the ubiquitination of p53. Cell 75, 495-505 (1993).

61. White, E. A. et al. Comprehensive analysis of host cellular interactions with human papillomavirus E6 proteins identifies new $\mathrm{E} 6$ binding partners and reflects viral diversity J. Virol 86, 13174-13186 (2012).

62. Tomaic, V. Functional roles of E6 and E7 oncoproteins in HPV-induced malignancies at diverse anatomical sites. Cancers 8, 95 (2016).

63. Eckhardt, M. et al. Multiple routes to oncogenesis are promoted by the human papillomavirus-host protein network. Cancer Discov. 8, 1474-1489 (2018).

64. Dyson, N., Howley, P. M., Munger, K. \& Harlow, E. The human papilloma virus-16 E7 oncoprotein is able to bind to the retinoblastoma gene product. Science 243, 934-937 (1989).

65. White, E. A et al. Systematic identification of interactions between host cell proteins and E7 oncoproteins from diverse human papillomaviruses. Proc. Natl Acad. Sci. USA 109, E260-E267 (2012).

66. Venuti, A. et al. Papillomavirus E5: the smallest oncoprotein with many functions. Mol. Cancer 10 140 (2011).

67. Estevao, D., Costa, N. R., Gil da Costa, R. M. \& Medeiros, R. Hallmarks of HPV carcinogenesis: the role of E6, E7 and E5 oncoproteins in cellular malignancy. Biochim. Biophys. Acta Gene Regul. Mech. 1862, 153-162 (2019).

68. Califano, J. et al. Genetic progression model for head and neck cancer: implications for field cancerization. Cancer Res. 56, 2488-2492 (1996). This paper provides the first genetic progression model for HNSCC.

69. Lui, V. W. et al. Frequent mutation of the PI3K pathway in head and neck cancer defines predictive biomarkers. Cancer Discov. 3, 761-769 (2013).

70. Nichols, A. C. et al. High frequency of activating PIK3CA mutations in human papillomavirus-positive oropharyngeal cancer. JAMA Otolaryngol. Head Neck Surg. 139, 617-622 (2013).

71. Agrawal, $\mathrm{N}$. et al. Exome sequencing of head and neck squamous cell carcinoma reveals inactivating mutations in NOTCH1. Science 333, 1154-1157 (2011). This was one of the first papers to describe NOTCH1 mutations in HNSCC.

72. Stransky, N. et al. The mutational landscape of head and neck squamous cell carcinoma. Science 333 1157-1160 (2011)

This was one of the first papers to report whole-exome sequencing in HNSCC.

73. Alsahafi, E. et al. Clinical update on head and neck cancer: molecular biology and ongoing challenges. Cell Death Dis. 10, 540 (2019).

74. Rocco, J. W. \& Ellisen, L. W. p63 and p73: life and death in squamous cell carcinoma. Cell Cycle 5 936-940 (2006)

75. Rocco, J. W., Leong, C. O., Kuperwasser, N., DeYoung, M. P. \& Ellisen, L. W. p63 mediates survival in squamous cell carcinoma by suppression of $\mathrm{p} 73$ dependent apoptosis. Cancer Cell 9, 45-56 (2006)

76. Yang, X. et al. $\Delta$ Np63 versatilely regulates a broad NF-kB gene program and promotes squamous epithelial proliferation, migration, and inflammation. Cancer Res. 71, 3688-3700 (2011).

77. Lu, H. et al. TNF- $\alpha$ promotes c-REL/ANp63alpha interaction and TAp73 dissociation from key genes that mediate growth arrest and apoptosis in head and neck cancer. Cancer Res. 71, 6867-6877 (2011).

78. $\mathrm{Si}, \mathrm{H}$. et al. TNF- $\alpha$ modulates genome-wide redistribution of $\triangle N p 63 \alpha / T A p 73$ and NF-kB CREL interactive binding on TP53 and AP- 1 motifs to promote an oncogenic gene program in squamous cancer. Oncogene 35, 5781-5794 (2016).

79. Lu, H. et al. CK2 phosphorylates and inhibits TAp73 tumor suppressor function to promote expression of cancer stem cell genes and phenotype in head and neck cancer. Neoplasia 16, 789-800 (2014).

80. Hu, Z. et al. Polo-like kinase 2 acting as a promoter in human tumor cells with an abundance of TAp73. Onco Targets Ther. 8, 3475-3488 (2015)

81. Foy, J. P. et al. New DNA methylation markers and global DNA hypomethylation are associated with oral cancer development. Cancer Prev. Res. 8, 1027-1035 (2015).

82. Viswanathan, M., Tsuchida, N. \& Shanmugam, G. Promoter hypermethylation profile of tumor-associated genes p16, p15, hMLH1, MGMT and E-cadherin in oral squamous cell carcinoma. Int. J. Cancer 105 41-46 (2003)

83. Ha, P. K. \& Califano, J. A. Promoter methylation and inactivation of tumour-suppressor genes in ora squamous-cell carcinoma. Lancet Oncol. 7, 77-82 (2006).

84. Rubin Grandis, J. et al. Levels of TGF-alpha and EGFR protein in head and neck squamous cell carcinoma and patient survival. J. Natl Cancer Inst. 90, 824-832 (1998)

85. Zhu, $X$ et al. Prognostic role of epidermal growth factor receptor in head and neck cancer: a meta-analysis. J. Surg. Oncol. 108, 387-397 (2013).

86. Madoz-Gurpide, J. et al. Activation of MET pathway predicts poor outcome to cetuximab in patients with recurrent or metastatic head and neck cancer. J. Trans/ Med. 13, 282 (2015).

87. Arnold, L., Enders, J. \& Thomas, S. M. Activated HGF-c-Met axis in head and neck cancer. Cancers $\mathbf{9}$, 169 (2017).

88. Karakasheva, T A et al. IL-6 mediates cross-talk between tumor cells and activated fibroblasts in the tumor microenvironment. Cancer Res. 78, 4957-4970 (2018).

89. Tsai, M. S., Chen, W. C., Lu, C. H. \& Chen, M. F. The prognosis of head and neck squamous cell carcinoma related to immunosuppressive tumor microenvironment regulated by IL-6 signaling. Oral. Oncol. 91 , 47-55 (2019).

90. Marsit, C. J., Black, C. C., Posner, M. R. \& Kelsey, K. T. A genotype-phenotype examination of cyclin D1 on risk and outcome of squamous cell carcinoma of the head and neck. Clin. Cancer Res. 14, 2371-2377 (2008)

91. Wang, Z., Valera, J. C., Zhao, X., Chen, Q. \& Gutkind, J. S. mTOR co-targeting strategies for head and neck cancer therapy. Cancer Metastasis Rev. 36, 491-502 (2017)

92. Squarize, C. H. et al. PTEN deficiency contributes to the development and progression of head and neck cancer. Neoplasia 15, 461-471 (2013).

93. Geiger, J. L., Grandis, J. R. \& Bauman, J. E. The STAT3 pathway as a therapeutic target in head and neck cancer: barriers and innovations. Oral. Oncol. $\mathbf{5 6}$ 84-92 (2016).

94. Johnson, D. E., O’Keefe, R. A. \& Grandis, J. R. Targeting the IL-6/JAK/STAT3 signalling axis in cancer. Nat. Rev. Clin. Oncol. 15, 234-248 (2018).

95. Lui, V. W. et al. Frequent mutation of receptor protein tyrosine phosphatases provides a mechanism for STAT3 hyperactivation in head and neck cancer. Proc. Natl Acad. Sci. USA 111, 1114-1119 (2014).

96. Peyser, N. D. et al. Loss-of-function PTPRD mutations lead to increased STAT3 activation and sensitivity to STAT3 inhibition in head and neck cancer. PLOS ONE 10, e0135750 (2015).

97. Alamoud, K. A. \& Kukuruzinska, M. A. Emerging insights into Wnt/ $\beta$-catenin signaling in head and neck cancer. J. Dent. Res. 97, 665-673 (2018).

98. Augsten, M. Cancer-associated fibroblasts as another polarized cell type of the tumor microenvironment. Front. Oncol. 4, 62 (2014).

99. Canning, M. et al. Heterogeneity of the head and neck squamous cell carcinoma immune landscape and its impact on immunotherapy. Front. Cell Dev. Biol. 7, 52 (2019).

100. Peltanova, B., Raudenska, M. \& Masarik, M. Effect of tumor microenvironment on pathogenesis of the head and neck squamous cell carcinoma: a systematic review. Mol. Cancer 18, 63 (2019).

101. Marsh, D. et al. Stromal features are predictive of disease mortality in oral cancer patients. J. Pathol. 223, 470-481 (2011).

102. Amit, M. et al. Loss of $\mathrm{p} 53$ drives neuron reprogramming in head and neck cancer. Nature 578, 449-454 (2020). 
103. Partlova, S. et al. Distinct patterns of intratumoral immune cell infiltrates in patients with HPV-associated compared to non-virally induced head and neck squamous cell carcinoma. Oncoimmunology 4 , e965570 (2015).

104. Mandal, R. et al. The head and neck cancer immune landscape and its immunotherapeutic implications. JCI Insight 1, e89829 (2016).

105. Chen, Y. P. et al. Identification and validation of novel microenvironment-based immune molecular subgroups of head and neck squamous cell carcinoma: implications for immunotherapy. Ann. Oncol. 30, 68-75 (2019)

106. Brooks, J. M. et al. Development and validation of a combined hypoxia and immune prognostic classifier for head and neck cancer. Clin. Cancer Res. 25 , 5315-5328 (2019).

107. Feng, B. et al. Integrative analysis of multi-omics data identified EGFR and PTCS2 as key nodes in a gene regulatory network related to immune phenotypes in head and neck cancer. Clin. Cancer Res. 26, 3616-3628 (2020)

108. Nguyen, N. et al. Tumor infiltrating lymphocytes and survival in patients with head and neck squamous cell carcinoma. Head Neck 38, 1074-1084 (2016)

109. Fang, J. et al. Prognostic significance of tumor infiltrating immune cells in oral squamous cell carcinoma. BMC Cancer 17, 375 (2017)

110. Whiteside, T. L. Immunobiology of head and neck cancer. Cancer Metastasis Rev. 24, 95-105 (2005)

111. Ward, M. J. et al. Tumour-infiltrating lymphocytes predict for outcome in HPV-positive oropharyngeal cancer. Br. J. Cancer 110, 489-500 (2014).

112. Costa, N. L. et al. Tumor-associated macrophages and the profile of inflammatory cytokines in oral squamous cell carcinoma. Oral. Oncol. 49, 216-223 (2013).

113. Ferris, R. L., Hunt, J. L. \& Ferrone, S. Human leukocyte antigen (HLA) class I defects in head and neck cancer: molecular mechanisms and clinical significance. Immunol. Res. 33, 113-133 (2005)

114. Ferris, R. L., Whiteside, T. L. \& Ferrone, S. Immune escape associated with functional defects in antigen-processing machinery in head and neck cancer. Clin. Cancer Res. 12, 3890-3895 (2006)

115. Grabowska, A. K. $\&$ Riemer, A. B. The invisible enemy - how human papillomaviruses avoid recognition and clearance by the host immune system. Open Virol. J. 6, 249-256 (2012)

116. DiMaio, D. \& Petti, L. M. The E5 proteins. Virology 445, 99-114 (2013)

117. Karim, R. et al. Human papillomavirus (HPV) upregulates the cellular deubiquitinase $\mathrm{UCHL} 1$ to suppress the keratinocyte's innate immune response. PLoS Pathog. 9, e1003384 (2013)

118. Gu, Z., Shi, W., Zhang, L., Hu, Z. \& Xu, C. USP19 suppresses cellular type I interferon signaling by targeting TRAF3 for deubiquitination. Future Microbiol. 12, 767-779 (2017)

119. Sorensen, B. S. et al. Radiosensitivity and effect of hypoxia in HPV positive head and neck cancer cells. Radiother. Oncol. 108, 500-505 (2013).

120. Brizel, D. M., Sibley, G. S., Prosnitz, L. R., Scher, R. L. $\&$ Dewhirst, M. W. Tumor hypoxia adversely affects the prognosis of carcinoma of the head and neck. Int. J. Radiat. Oncol. Biol. Phys. 38, 285-289 (1997).

121. Swartz, J. E. et al. Poor prognosis in human papillomavirus-positive oropharyngeal squamous cell carcinomas that overexpress hypoxia inducible factor-1 a. Head Neck 38, 1338-1346 (2016).

122. Gottgens, E. L., Ostheimer, C., Span, P. N., Bussink, J. \& Hammond, E. M. HPV, hypoxia and radiation response in head and neck cancer. Br. J. Radiol. 92 1093 (2019).

123. Nordsmark, M. et al. Prognostic value of tumor oxygenation in 397 head and neck tumors after primary radiation therapy. An international multi-center study. Radiother. Oncol. 77, 18-24 (2005).

124. Linge, A. et al. Low cancer stem cell marker expression and low hypoxia identify good prognosis subgroups in HPV(-) HNSCC after postoperative radiochemotherapy: a multicenter study of the DKTK-ROG. Clin. Cancer Res. 22, 2639-2649 (2016)

125. Bornigen, D. et al. Alterations in oral bacterial communities are associated with risk factors for oral and oropharyngeal cancer. Sci. Rep. 7, 17686 (2017).

126. Mager, D. L. et al. The salivary microbiota as a diagnostic indicator of oral cancer: a descriptive non-randomized study of cancer-free and oral squamous cell carcinoma subjects. J. Trans/ Med. 3 27 (2005)
127. Banerjee, S. et al. Microbial signatures associated with oropharyngeal and oral squamous cell carcinomas. Sci. Rep. 7, 4036 (2017)

128. Luukkaa, M. et al. Association between high collagenase-3 expression levels and poor prognosis in patients with head and neck cancer. Head Neck 28, 225-234 (2006)

129. Patel, B. P., Shah, S. V., Shukla, S. N., Shah, P. M. \& Patel, P. S. Clinical significance of MMP-2 and MMP-9 in patients with oral cancer. Head Neck 29, 564-572 (2007).

130. Viros, D. et al. Prognostic role of MMP-9 expression in head and neck carcinoma patients treated with radiotherapy or chemoradiotherapy. Oral. Oncol. 49, 322-325 (2013)

131. Samanna, V., Ma, T., Mak, T. W., Rogers, M. \& Chellaiah, M. A. Actin polymerization modulates CD44 surface expression, MMP-9 activation, and osteoclast function. J. Cell Physiol. 213, 710-720 (2007).

132. Sterz, C. M. et al. A basal-cell-like compartment in head and neck squamous cell carcinomas represents the invasive front of the tumor and is expressing MMP-9 Oral Oncol 46, 116-122 (2010).

133. Nijkamp, M. M. et al. Expression of E-cadherin and vimentin correlates with metastasis formation in head and neck squamous cell carcinoma patients. Radiother. Oncol. 99, 344-348 (2011).

134. Yang, M. H. et al. Direct regulation of TWIST by HIF-1 alpha promotes metastasis. Nat. Cell Biol. 10 295-305 (2008)

135. Zhang, Z., Filho, M. S. \& Nor, J. E. The biology of head and neck cancer stem cells. Oral. Oncol. 48 1-9 (2012).

136. Williams, E. D., Gao, D., Redfern, A. \& Thompson, E. W. Controversies around epithelial-mesenchymal plasticity in cancer metastasis. Nat. Rev. Cancer 19, 716-732 (2019).

137. Puram, S. V. et al. Single-cell transcriptomic analysis of primary and metastatic tumor ecosystems in head and neck cancer. Cell 171, 1611-1624 e24 (2017).

138. Nirmala, J. G. \& Lopus, M. Cell death mechanisms in eukaryotes. Cell Biol. Toxicol. 36, 145-164 (2020).

139. Zeng, Q. et al. Hepatocyte growth factor inhibits anoikis in head and neck squamous cell carcinoma cells by activation of ERK and Akt signaling independent of NFkB. J. Biol. Chem. 277, 25203-25208 (2002)

140. Neiva, K. G. et al. Cross talk initiated by endothelial cells enhances migration and inhibits anoikis of squamous cell carcinoma cells through STAT3/Akt/ERK signaling. Neoplasia 11, 583-593 (2009).

141. Huang, S. H. et al. Refining American Joint Committee on Cancer/Union for International Cancer Control TNM stage and prognostic groups for human papillomavirus-related oropharyngeal carcinomas. J. Clin. Oncol. 33, 836-845 (2015). This paper refines the TNM staging for HPV-positive HNSCC.

142. Hashibe, M. et al. Interaction between tobacco and alcohol use and the risk of head and neck cancer: pooled analysis in the International Head and Neck Cancer Epidemiology Consortium. Cancer Epidemiol. Biomarkers Prev. 18, 541-550 (2009).

143. Taberna, M. et al. Human papillomavirus-related oropharyngeal cancer. Ann. Oncol. 28, 2386-2398 (2017).

144. Fu, T. S., Foreman, A., Goldstein, D. P. \& de Almeida, J. R. The role of transoral robotic surgery, transoral laser microsurgery, and lingual tonsillectomy in the identification of head and neck squamous cell carcinoma of unknown primary origin: a systematic review. J. Otolaryngol. Head Neck Surg. 45, 28 (2016).

145. Meccariello, G. et al. The emerging role of trans-ora robotic surgery for the detection of the primary tumour site in patients with head-neck unknown primary cancers: a meta-analysis. Auris Nasus Larynx 46, 663-671 (2019).

146. Chen, Y. P. et al. Nasopharyngeal carcinoma. Lance 394, 64-80 (2019).

147. Pynnonen, M. A. et al. Clinical practice guideline: evaluation of the neck mass in adults. Otolaryngol. Head Neck Surg. 157, S1-S30 (2017).

148. Yi, C. H., Jim Zhai, Q. \& Wang, B. Y. Updates on immunohistochemical and molecular markers in selected head and neck diagnostic problems Arch. Pathol. Lab. Med. 141, 1214-1235 (2017)

149. Lewis, J. S. Jr. et al. Human papillomavirus testing in head and neck carcinomas: guideline from the College of American Pathologists. Arch. Pathol. Lab. Med. 142, 559-597 (2018).
150. Ang, K. K. et al. Human papillomavirus and survival of patients with oropharyngeal cancer. N. Engl. J. Med. 363, 24-35 (2010). This paper describes the distinct survival characteristics of HPV-positive HNSCC.

151. Edge, S. et al. (eds) AJCC Cancer Staging Manual 7th edn (Springer, 2010)

152. Amin, M. B. et al. (eds). AJCC Cancer Staging Manual 8th edn (Springer, 2017).

153. Lydiatt, W., O'Sullivan, B. \& Patel, S. Major changes in head and neck staging for 2018. Am. Soc. Clin. Oncol. Educ. Book. 38, 505-514 (2018).

154. van Gysen, K. et al. Validation of the 8(th) edition UICC/AJCC TNM staging system for HPV associated oropharyngeal cancer patients managed with contemporary chemo-radiotherapy. BMC Cancer 19 , 674 (2019).

155. Wurdemann, N. et al. Prognostic impact of AJCC/UICC 8th edition new staging rules in oropharyngeal squamous cell carcinoma. Front. Oncol. 7, 129 (2017).

156. Lee, V. H. et al. The addition of pretreatment plasma Epstein-Barr virus DNA into the eighth edition of nasopharyngeal cancer TNM stage classification Int. J. Cancer 144, 1713-1722 (2019).

157. Guo, R et al Proposed modifications and incorporation of plasma Epstein-Barr virus DNA improve the TNM staging system for Epstein-Barr virus-related nasopharyngeal carcinoma. Cancer 125 79-89 (2019)

158. Pfister, D. G. et al. Head and neck cancers, version 2.2020, NCCN Clinical Practice Guidelines in Oncology. J. Natl Compr. Canc Netw. 18, 873-898 (2020).

159. Coca-Pelaz, A. et al. The risk of second primary tumors in head and neck cancer: a systematic review. Head Neck 42, 456-466 (2020).

This paper underscores the high risk of developing second primary tumors in carcinogen-associated HNSCC.

160. U.S. Department of Health and Human Services. Smoking Cessation: a Report of the Surgeon General. U.S. Department of Health and Human Services https://www.hhs.gov/sites/default/files/2020 cessation-sgr-full-report.pdf (2020)

161. World Health Organization. Tackling NCDs: 'best buys' and other recommended interventions for the prevention and control of noncommunicable diseases. WHO https://apps.who.int/iris/handle/10665/259232 (2017).

162. Mehrtash, H. et al. Defining a global research and policy agenda for betel quid and areca nut Lancet Oncol. 18, e767-e775 (2017).

163. Herrero, R. et al. Reduced prevalence of oral human papillomavirus (HPV) 4 years after bivalent HPV vaccination in a randomized clinical trial in Costa Rica. PLOS ONE 8, e68329 (2013).

164. Hirth, J. M., Chang, M., Resto, V. A. \& Group, H. P. V. S Prevalence of oral human papillomavirus by vaccination status among young adults (18-30 years old). Vaccine 35, 3446-3451 (2017)

165. Chaturvedi, A. K. et al. Effect of prophylactic human papillomavirus (HPV) vaccination on oral HPV infections among young adults in the United States. J. Clin. Oncol. 36, 262-267 (2018). This paper provides an early insight into the impact of vaccination on HPV oral infection.

166. Meites, E., Kempe, A. \& Markowitz, L. E. Use of a 2-dose schedule for human papillomavirus vaccination - updated recommendations of the Advisory Committee on Immunization Practices. Morb. Mortal. Wkly Rep. 65, 1405-1408 (2016).

167. Meites, E et al. Human papillomavirus vaccination for adults: updated recommendations of the Advisory Committee on Immunization Practices. Morb. Mortal. Wkly Rep. 68, 698-702 (2019)

168. Napier, S. S. $\&$ Speight, P. M. Natural history of potentially malignant oral lesions and conditions: an overview of the literature $J$ Oral Pathol. Med. 37, 1-10 (2008)

169. Lee, J. J. et al. Predicting cancer development in oral leukoplakia: ten years of translational research Clin. Cancer Res. 6, 1702-1710 (2000).

170. Hong, W. K. et al. 13-cis-retinoic acid in the treatment of oral leukoplakia. N. Engl. J. Med. 315, 1501-1505 (1986).

171. Hong, W. K. et al. Prevention of second primary tumors with isotretinoin in squamous-cell carcinoma of the head and neck. N. Engl. J. Med. 323, 795-80 (1990).

172. Rosin, M. P. et al. Use of allelic loss to predict malignant risk for low-grade oral epithelial dysplasia. Clin. Cancer Res. 6, 357-362 (2000). 
173. Garland, L. L. et al. Effect of intermittent versus continuous low-dose aspirin on nasal epithelium gene expression in current smokers: a randomized, double-blinded trial. Cancer Prev. Res. 12, 809-820 (2019).

174. Vitale-Cross, L. et al. Metformin prevents the development of oral squamous cell carcinomas from carcinogen-induced premalignant lesions. Cancer Prev. Res. 5, 562-573 (2012)

175. Bauman, J. E. et al. Prevention of carcinogen-induced oral cancer by sulforaphane. Cancer Prev. Res. 9 , 547-557 (2016).

176. Hu, L. et al. Gene targets of sulforaphane in head and neck squamous cell carcinoma. Mol. Med. Rep. 20, 5335-5344 (2019)

177. Lee, N. C. J. et al. Patterns of failure in high-metastatic node number human papillomavirus-positive oropharyngeal carcinoma. Oral. Oncol. 85, 35-39 (2018).

178. Lyhne, N. M. et al. The DAHANCA 6 randomized trial: effect of 6 vs 5 weekly fractions of radiotherapy in patients with glottic squamous cell carcinoma. Radiother. Oncol. 117, 91-98 (2015).

179. Bledsoe, T. J. et al. Hypofractionated radiotherapy for patients with early-stage glottic cancer: patterns of care and survival. J. Natl Cancer Inst. https://doi.org 10.1093/jnci/djx042 (2017).

180. Weinstein, G. S. et al. Transoral robotic surgery: a multicenter study to assess feasibility, safety, and surgical margins. Laryngoscope 122, 1701-1707 (2012)

181. Forastiere, A. A. et al. Use of larynx-preservation strategies in the treatment of laryngeal cancer: American Society of Clinical Oncology clinical practice guideline update. J. Clin. Oncol. 36, 1143-1169 (2018)

182. D'Cruz, A. K. et al. Elective versus therapeutic neck dissection in node-negative oral cancer. N. Engl. J. Med. 373, 521-529 (2015).

183. Forastiere, A. A. et al. Concurrent chemotherapy and radiotherapy for organ preservation in advanced laryngeal cancer. N. Engl. J. Med. 349, 2091-2098 (2003).

184. Cooper, J. S. et al. Postoperative concurrent radiotherapy and chemotherapy for high-risk squamous-cell carcinoma of the head and neck. N. Engl. J. Med. 350, 1937-1944 (2004)

185. Bernier, J. et al. Postoperative irradiation with or without concomitant chemotherapy for locally advanced head and neck cancer. N. Engl. J. Med. 350, 1945-1952 (2004).

186. Forastiere, A. A. et al. Long-term results of RTOG 91-11: a comparison of three nonsurgical treatment strategies to preserve the larynx in patients with locally advanced larynx cancer. J. Clin. Oncol. 31 , 845-852 (2013)

187. Kelly, J. R. et al. Upfront surgery versus definitive chemoradiotherapy in patients with human papillomavirus-associated oropharyngeal squamous cell cancer. Oral. Oncol. 79, 64-70 (2018).

188. Kann, B. H. et al. Pretreatment identification of head and neck cancer nodal metastasis and extranodal extension using deep learning neural networks. Sci. Rep. 8, 14036 (2018).

189. Lowe, V. J. et al. Multicenter trial of [(18)F] fluorodeoxyglucose positron emission tomography/ computed tomography staging of head and neck cancer and negative predictive value and surgical impact in the N0 neck: results from ACRIN 6685. J. Clin. Oncol. 37, 1704-1712 (2019). This study highlights the role of PET-CT scanning in $\mathrm{HNSCC}$.

190. Adelstein, D. J. et al. An intergroup phase III comparison of standard radiation therapy and two schedules of concurrent chemoradiotherapy in patients with unresectable squamous cell head and neck cancer. J. Clin. Oncol. 21, 92-98 (2003).

191. Bauml, J. M. et al. Cisplatin every 3 weeks versus weekly with definitive concurrent radiotherapy for squamous cell carcinoma of the head and neck. J. Natl Cancer Inst. 111, 490-497 (2019).

192. Worden, F. P. et al. Chemoselection as a strategy for organ preservation in patients with T4 laryngeal squamous cell carcinoma with cartilage invasion. Laryngoscope 119, 1510-1517 (2009).

193. Blanchard, P. et al. Chemotherapy and radiotherapy in nasopharyngeal carcinoma: an update of the MAC-NPC meta-analysis. Lancet Oncol. 16, 645-655 (2015).

194. Zhang, Y. et al. Gemcitabine and cisplatin induction chemotherapy in nasopharyngeal carcinoma. N. Engl. J. Med. 381, 1124-1135 (2019).
195. Chan, A. T. C. et al. Analysis of plasma Epstein-Barr virus DNA in nasopharyngeal cancer after chemoradiation to identify high-risk patients for adjuvant chemotherapy: a randomized controlled trial. J. Clin. Oncol. 36, 3091-3100 (2018).

196. Trotti, A. et al. TAME: development of a new method for summarising adverse events of cancer treatment by the Radiation Therapy Oncology Group. Lancet Oncol. 8, 613-624 (2007).

197. Haddad, R. et al. Induction chemotherapy followed by concurrent chemoradiotherapy (sequential chemoradiotherapy) versus concurrent chemoradiotherapy alone in locally advanced head and neck cancer (PARADIGM): a randomised phase 3 trial. Lancet Oncol. 14, 257-264 (2013).

198. Cohen, E. E. et al. Phase III randomized trial of induction chemotherapy in patients with $\mathrm{N} 2$ or N3 locally advanced head and neck cancer. J. Clin. Oncol. 32, 2735-2743 (2014)

199. Posner, M. R. et al. Cisplatin and fluorouracil alone or with docetaxel in head and neck cancer. N. Engl. J. Med. 357, 1705-1715 (2007).

200. Kowalski, L. P. et al. COVID-19 pandemic: effects and evidence-based recommendations for otolaryngology and head and neck surgery practice. Head Neck 42, 1259-1267 (2020).

201. Fakhry, C. et al. Improved survival of patients with human papillomavirus-positive head and neck squamous cell carcinoma in a prospective clinical trial J. Natl Cancer Inst. 100, 261-269 (2008)

202. Marur, S. et al. E1308: phase II trial of induction chemotherapy followed by reduced-dose radiation and weekly cetuximab in patients with HPV-associated resectable squamous cell carcinoma of the oropharynx - ECOG-ACRIN cancer research group. J. Clin. Oncol. 35, 490-497 (2017)

203. Chen, A. M. et al. Reduced-dose radiotherapy for human papillomavirus-associated squamous-cell carcinoma of the oropharynx: a single-arm, phase 2 study. Lancet Oncol. 18, 803-811 (2017).

204. Lee, A. W. et al. Retrospective analysis of patients with nasopharyngeal carcinoma treated during 1976-1985: survival after local recurrence. Int. J. Radiat. Oncol. Biol. Phys. 26, 773-782 (1993).

205. Fakhry, C. et al. Human papillomavirus and overall survival after progression of oropharyngeal squamous cell carcinoma. J. Clin. Oncol. 32, 3365-3373 (2014).

206. Saada-Bouzid, E. et al. Hyperprogression during anti-PD-1/PD-L1 therapy in patients with recurrent and/or metastatic head and neck squamous cel carcinoma. Ann. Oncol. 28, 1605-1611 (2017).

207. Saleh, K. et al. Response to salvage chemotherapy after progression on immune checkpoint inhibitors in patients with recurrent and/or metastatic squamous cell carcinoma of the head and neck. Eur. J. Cancer 121, 123-129 (2019)

208. Puzanov, I. et al. Managing toxicities associated with immune checkpoint inhibitors: consensus recommendations from the Society for Immunotherapy of Cancer (SITC) Toxicity Management Working Group. J. Immunother. Cancer 5, 95 (2017).

209. Vermorken, J. B. et al. Platinum-based chemotherapy plus cetuximab in head and neck cancer. N. Engl. J. Med. 359, 1116-1127 (2008)

210. Cohen, E. E. W. et al. Pembrolizumab versus methotrexate, docetaxel, or cetuximab for recurrent or metastatic head-and-neck squamous cell carcinoma (KEYNOTE-040): a randomised, open-label, phase 3 study. Lancet 393, 156-167 (2019).

211. Mehra, R. et al. Efficacy and safety of pembrolizumab in recurrent/metastatic head and neck squamous cell carcinoma: pooled analyses after long-term follow-up in KEYNOTE-012. Br. J. Cancer 119, 153-159 (2018).

212. Haddad, R. et al. Nivolumab treatment beyond RECIST-defined progression in recurrent or metastatic squamous cell carcinoma of the head and neck in CheckMate 141: a subgroup analysis of a randomized phase 3 clinical trial. Cancer 125, 3208-3218 (2019).

213. Taylor, M. H. et al. Phase IB/II trial of lenvatinib plus pembrolizumab in patients with advanced renal cell carcinoma, endometrial cancer, and other selected advanced solid tumors. J. Clin. Oncol. 38, 1154-1163 (2020).

214. Verdonck-de Leeuw, I. M. et al. The course of health-related quality of life in head and neck cancer patients treated with chemoradiation: a prospective cohort study. Radiother. Oncol. 110, 422-428 (2014).
215. Rettig, E. M. et al. Health-related quality of life before and after head and neck squamous cell carcinoma: analysis of the Surveillance, Epidemiology, and End Results-Medicare Health Outcomes Survey linkage Cancer 122, 1861-1870 (2016)

216. Quinten, C. et al. Baseline quality of life as a prognostic indicator of survival: a meta-analysis of individual patient data from EORTC clinical trials. Lancet Oncol. 10, 865-871 (2009).

217. Rogers, S. N. et al. Quality of life, cognitive, physical and emotional function at diagnosis predicts head and neck cancer survival: analysis of cases from the head and neck 5000 study. Eur. Arch. Otorhinolaryngol. 277, 1515-1523 (2020)

218. Hammerlid, E., Silander, E., Hornestam, L. \& Sullivan, M. Health-related quality of life three years after diagnosis of head and neck cancer-a longitudinal study. Head Neck 23, 113-125 (2001).

219. Mehanna, H. M. \& Morton, R. P. Deterioration in quality-of-life of late (10-year) survivors of head and neck cancer. Clin. Otolaryngol. 31, 204-211 (2006).

220. Verdonck-de Leeuw, I. M., van Nieuwenhuizen, A $\&$ Leemans, C. R. The value of quality-of-life questionnaires in head and neck cancer. Curr. Opin. Otolaryngol. Head Neck Surg. 20, 142-147 (2012).

221. Singer S et al International validation of the revised European Organisation for Research and Treatment of Cancer Head and Neck Cancer Module, the EORTC QLQ-HN43: phase IV. Head Neck 41, 1725-1737 (2019).

222. List, M. A. et al. The performance status scale for head and neck cancer patients and the functional assessment of cancer therapy-head and neck scale. A study of utility and validity. Cancer 77, 2294-2301 (1996).

223. Nekhlyudov, L. et al. Head and neck cancer survivorship care guideline: American Society of Clinical Oncology clinical practice guideline endorsement of the American Cancer Society guideline. J. Clin. Oncol. 35, 1606-1621 (2017).

224. Ringash, J. et al. Head and neck cancer survivorship: learning the needs, meeting the needs. Semin. Radiat. Oncol. 28, 64-74 (2018)

225. Bonomo, P. et al. Quality assessment in supportive care in head and neck cancer. Front. Oncol. 9, 926 (2019).

226. Rinkel, R. N. et al. Prevalence of swallowing and speech problems in daily life after chemoradiation for head and neck cancer based on cut-off scores of the patient-reported outcome measures SWAL-QOL and SHI. Eur. Arch. Otorhinolaryngol. 273 1849-1855 (2016)

227. Kraaijenga, S. A. et al. Assessment of voice, speech and related quality of life in advanced head and neck cancer patients 10-years+ after chemoradiotherapy. Oral. Oncol. 55, 24-30 (2016).

228. Hutcheson, K. A. et al. Two-year prevalence of dysphagia and related outcomes in head and neck cancer survivors: an updated SEER-Medicare analysis. Head Neck 41, 479-487 (2019)

229. Bjordal, K. \& Bottomley, A. Making advances in quality of life studies in head and neck cancer. Int. J. Radiat. Oncol. Biol. Phys. 97, 659-661 (2017).

230. Dawson, C. et al. Patient advocacy in head and neck cancer: realities, challenges and the role of the multi-disciplinary team. Clin. Otolaryngol. 45 437-444 (2020)

231. van der Hout, A. et al. Role of eHealth application Oncokompas in supporting self-management of symptoms and health-related quality of life in cancer survivors: a randomised, controlled trial. Lancet Oncol. 21, 80-94 (2020).

232. Parke, S. C. et al. Identifying gaps in research on rehabilitation for patients with head and neck cancer: a scoping review. Arch. Phys. Med. Rehabil. 100, 2381-2388 (2019).

233. Kampshoff, C. S., Verdonck-de Leeuw, I. M. van Oijen, M. G., Sprangers, M. A. \& Buffart, L. M. Ecological momentary assessments among patients with cancer: a scoping review. Eur. J. Cancer Care $\mathbf{2 8}$ e13095 (2019).

234. David, J. M. et al. Treatment at high-volume facilities and academic centers is independently associated with improved survival in patients with locally advanced head and neck cancer. Cancer 123. 3933-3942 (2017).

235. Wuthrick, E. J. et al. Institutional clinical trial accrual volume and survival of patients with head and neck cancer. J. Clin. Oncol. 33, 156-164 (2015). 
236. Bossi, P. et al. Impact of treatment expertise on the outcome of patients with head and neck cancer treated within 6 randomized trials. Head Neck 40 2648-2656 (2018)

237. Ellington, T. D. et al. Trends in incidence of cancers of the oral cavity and pharynx - United States 2007-2016. Morb. Mortal. Wkly Rep. 69, 433-438 (2020).

238. Sonawane, K. et al. Oral human papillomavirus infection: differences in prevalence between sexes and concordance with genital human papillomavirus infection, NHANES 2011 to 2014. Ann. Intern. Med. 167, 714-724 (2017).

239. [No authors listed] Tipifarnib targets HRAS-mutant cancers. Cancer Discov. 9, 1637-1638 (2019).

240. Elkabets, M. et al. AXL mediates resistance to $\mathrm{PI} 3 \mathrm{Ka}$ inhibition by activating the EGFR/PKC/mTOR axis in head and neck and esophageal squamous cell carcinomas. Cancer Cell 27, 533-546 (2015).

241. Merino, D. M. et al. Establishing guidelines to harmonize tumor mutational burden (TMB): in silico assessment of variation in TMB quantification across diagnostic platforms: phase I of the Friends of Cancer Research TMB Harmonization Project. J. Immunother. Cancer 8, e000147 (2020).

242. Castellanos, E. \& Baxi, S. S. Letting the GENIE out of its bottle: examining the potential of real-world clinicogenomic data. Cancer Discov. 10, 490-491 (2020).

243. Cillo, A R et al. Immune landscape of viral- and carcinogen-driven head and neck cancer. Immunity 52, 183-199.e9 (2020).

244. Li, H. et al. Proteomic characterization of head and neck cancer patient-derived xenografts. Mol. Cancer Res. 14, 278-286 (2016).

245. Karamboulas, C. et al. Patient-derived xenografts for prognostication and personalized treatment for head and neck squamous cell carcinoma. Cell Rep. 25, 1318-1331.e4 (2018)

246. Driehuis, E. et al. Oral mucosal organoids as a potential platform for personalized cancer therapy. Cancer Discov. 9, 852-871 (2019).

247. Wang, H. et al. Immune checkpoint inhibitor toxicity in head and neck cancer: from identification to management. Front. Pharmacol. 10, 1254 (2019).

248. Wang, Z. et al. Syngeneic animal models of tobacco-associated oral cancer reveal the activity of in situ anti-CTLA-4. Nat. Commun. 10, 5546 (2019).

249. Barry, K. C. et al. A natural killer-dendritic cell axis defines checkpoint therapy-responsive tumor microenvironments. Nat. Med. 24, 1178-1191 (2018)

250. Shah, F. D. et al. A review on salivary genomics and proteomics biomarkers in oral cancer. Indian. $J$. Clin. Biochem. 26, 326-334 (2011).

\section{Acknowledgements}

This work was supported by the following: NIH R35CA231998 (J.R.G.), U54CA209891 (J.R.G.) R01DE023685 (J.R.G and D.E.J), R01DE028289 (D.E.J. and J.R.G.), NET-QUBIC-Dutch Cancer Society grant VU 2013-5930 (C.R.L.), General Research Fund no. 17121616 and no. 14168517 (V.W.Y.L.), Research Impact Fund R4015 19F and R4017-18 (V.W.Y.L.), the Health and Medica Research Fund by the Food and Health Bureau, the Government of the Hong Kong Special Administrative Region no. 15160691 (V.W.Y.L.), University-Industry Collaboration Program UIM/329 by the Innovation and Technology Fund Hong Kong Government, Hong Kong SAR (V.W.Y.L.), the Hong Kong Cancer Fund, Hong Kong SAR (V.W.Y.L.), NIH/NC UG 1 CA2 42596 (J.E.B.), and NIH/NCI P30CA023074 (J.E.B.)

\section{Author contributions}

Introduction (J.R.G.); Epidemiology (V.W.Y.L.); Pathophysiology/mechanisms (D.E.J.); Diagnosis, screening and prevention (J.E.B.); Management (B.B.); Quality of life (C.R.L.); Outlook (D.E.J. and J.R.G.).

\section{Competing interest}

D.E.J. and J.R.G. are co-inventors of cyclic STAT3 decoy and have financial interests in STAT3 Therapeutics. STAT3 Therapeutics holds an interest in cyclic STAT3 decoy. B.B. has received honoraria for consulting from Merck and AstraZeneca. C.R.L. serves on the Advisory Board of Merck \& Co. and Rakuten Medical. V.W.Y.L. receives grant support from Lee's Pharmaceutical, Hong Kong Limited, via the University-Industry Collaboration Program (UIM/329; from the Innovation and Technology Fund, Hong Kong Government; in 2018-2020), and served as a scientific consultant for Novartis Pharmaceutical (Hong Kong) Limited (Oct 2015Oct 2016). J.E.B. serves as a scientific consultant to CUE Pharmaceuticals and AstraZeneca and has research grant support from the IST programmes of Aveo and Novartis.

\section{Peer review information}

Nature Reviews Disease Primers thanks C. Chung, C. Van Waes, A. Dietz, J. Hess, C. Bradford and the other, anonymous, reviewer(s) for their contribution to the peer review of this work.

\section{Publisher's note}

Springer Nature remains neutral with regard to jurisdictional claims in published maps and institutional affiliations.

Springer Nature or its licensor (e.g. a society or other partner) holds exclusive rights to this article under a publishing agreement with the author(s) or other rightsholder(s); author self-archiving of the accepted manuscript version of this article is solely governed by the terms of such publishing agreement and applicable law.

\section{RELATED LINKS}

Global Cancer Observatory (GLOBOCAN): https://gco.iarc.fr/ GLOBOCAN website mapping tool: https://gco.iarc.fr/today/ online-analysis-map

(c) Springer Nature Limited 2020, corrected publication 2023 